\title{
THE DIRICHLET PROBLEM FOR DOMAINS WITH MULTIPLE BOUNDARY POINTS*
}

\author{
BY \\ F. W. PERKINS \\ INTRODUCTION
}

Several years ago Professor Kellogg called my attention to the desirability of extending the theory of the Dirichlet problem so as to include the case in which the domain has multiple boundary points with boundary values depending upon the manner of approach. In the two-dimensional case conformal mapping may sometimes be used. Also, a paper by Perron $\dagger$ contains results related to this subject. It seems desirable, however, to develop a general theory for this extended form of the problem.

Professor Kellogg noted that a spatial analogue of Carathéodory’s $\ddagger$ theory of prime ends would be of value here, since this would render possible in some cases the definition of functions corresponding to barriers. $\$ \mathrm{He}$ communicated his ideas on this topic to me, and invited me to collaborate with him on the problem. Later he suggested that I develop the subject alone, a procedure which unfortunately was made necessary by his death. This paper contains the results of the ensuing study of the problem.

The discussion is formulated for a general finite domain of three-dimensional space, except in the case of a few theorems where special restrictions are imposed. It is readily seen that corresponding results are valid in the plane.

In Part I we introduce the notions of component and boundary element. These correspond to Carathéodory's ends and prime ends, respectively,

* Presented to the Society, December 26, 1933; received by the editors August 16, 1934, and in revised form, January 5, 1934.

$\dagger$ O. Perron, Eine neue Behandlung der ersten Randwertaufgabe für $\Delta u=0$, Mathematische Zeitschrift, vol. 18 (1923), pp. 42-55. See also N. Wiener, Note on a paper of O. Perron, Journal of Mathematics, and Physics of the Massachusetts Institute of Technology, vol. 4, No. 1 (January, 1925), p. $21 \mathrm{ff}$.

‡ C. Carathéodory, Über die Begrenzung einfach zusammenhängender Gebiete, Mathematische Annalen, vol. 73 (1913), pp. 323-370. I am indebted to Dr. Seidel for calling my attention to a group of papers containing a spatial generalization of this theory, though not of the type desired for the present problem: B. Kaufmann, Über die Berandung ebener und räumlicher Gebiete (Primendentheorie), Mathematische Annalen, vol. 103 (1930), pp. 70-144; Über die Struktur der Komplexe erster Ordnung in der Theorie der Primenden, ibid., vol. 106 (1932), pp. 308-333; Über die Bestimmung der Primenden durch reguläre Komplexe, ibid., vol. 106 (1932), pp. 334-342.

$\S$ See Theorem 24 of this paper. Professor Kellogg suggested the construction of a barrier by means of an infinite series, as is done here. 
though the analogy is not as close as that which I believe Professor Kellogg had in mind. Like the prime ends of Carathéodory's theory, boundary elements furnish a means of distinguishing between the various modes of approach to a multiple boundary point, which (for our purposes) may be defined as a point contained in more than one boundary element.

Part II is devoted largely to the study of functions of boundary elements. We introduce various concepts corresponding to familiar notions in the theory of functions of a real variable. We give in $\$ 2$ a theorem which is used later as a substitute for the Weierstrass polynomial approximation theorem.

In Part III we attack the Dirichlet problem. It is shown that the sequence solution of the generalized Dirichlet problem studied by Wiener* and by Kellogg $\dagger$ has a direct analogue in the new theory. The discussion here given corresponds quite closely to Kellogg's treatment of the earlier form of the problem, except that we confine our attention to finite domains. We also introduce the idea of a "pseudo-barrier" and discuss its properties briefly.

\section{COMPONENTS AND BOUNDARY ELEMENTS}

\section{Preliminary ideas}

In addition to a number of special propositions necessary in connection with the later theory, we include here explicit definitions of certain familiar concepts, in order to avoid ambiguity in our use of these terms.

DeFINITION 1. By a finite domain, or a domain $T$, we mean a proper $\ddagger$ bounded, open, connected $\$$ point set in a three-dimensional euclidean space.

Except in the case of a few propositions where special restrictions are desirable, we will find it convenient to think of the domain $T$ as chosen initially in an arbitrary fashion, and then held fast throughout the discussion.

* N. Wiener, Certain notions of potential theory, Journal of Mathematics and Physics, vol. 3, No. 1 (January, 1924), p. $24 \mathrm{ff}$.

† O. D. Kellogg, Foundations of Potential Theory, Berlin,1929, pp.322-326. (See also Proceedings of the American Academy, vol. 58 (1923), pp. 528, 529.)

$\ddagger$ A proper point set is one which contains at least one point. A proper subset of a given set is a proper set contained in the given set but not identical with it.

$\S$ A point set satisfying the other requirements will be called connected if, given any two points of the set, it is possible to join them by a continuous curve lying entirely in the set. By a continuous curve we mean a closed point set which with reference to some (and therefore any) Cartesian coordinate system, may be represented parametrically by equations of the form

$$
x=X(\theta), \quad y=Y(\theta), \quad z=Z(\theta), \quad 0 \leqq \theta \leqq 1,
$$

where the functions $X(\theta), Y(\theta)$, and $Z(\theta)$ are each defined and continuous on the interval $0 \leqq \theta \leqq 1$. The ends of the curve are the points corresponding to $\theta=0$ and $\theta=1$. It will be noted that we make no restriction that such a curve may not cross itself. 
DEFINITION 2. By a finite closed region $T^{\prime}$ we mean a point set which may be obtained by adjoining to a finite domain $T$ all points of its boundary, ${ }^{*} t$.

DEFINITION 3. Given any proper subset $E$ of the closed finite region $T^{\prime}=T+t$, the closed set comprising all boundary points of $E$ which are not in $t$, and all limit points of such boundary points, is called the auxiliary boundary of $E$.

DEFINITION 4. By a partial domain $G$ of $T$ we mean a finite domain having the following properties:

(1) The set $G$ is a proper subset of $T$.

(2) At least one point of $t$ is a boundary point of $G$.

(3) The auxiliary boundary, $g$, of $G$ contains only frontier points of $G$.

Definition 5. By a closed partial region $G^{\prime}$ of $T^{\prime}$ we mean a finite closed region which may be formed by adjoining to a partial domain $G$ of $T$ all boundary points of $G$.

THEOREM 1. The set of all points of $T$ which are interior points of a closed partial region $G^{\prime}$ of $T^{\prime}$ is identical with the partial domain $G$ of $T$ from which $G^{\prime}$ is formed. Moreover, the auxiliary boundaries of $G$ and $G^{\prime}$ are proper sets and are identical.

A point of $T$ interior to $G^{\prime}$ is not an exterior point of $G$; moreover it is not a limit point of points exterior to $G$, and so cannot be a frontier point of $G$. Hence such a point cannot be a boundary point of $G$, since all boundary points of $G$ are frontier points of $G$. Consequently a point of $T$ interior to $G^{\prime}$ is a point of $G$. Since a point of $G$ is a point of $T$ interior to $G^{\prime}$ we see that the first part of the theorem is true.

If the proper set $G$ had no boundary points except points of $t$, we could infer that every point of $T$ is a point of $G$, contrary to the definition of $G$. Since $T$ contains points not in $G$, it must contain a boundary point of $G$, and so the auxiliary boundary of $G$ is a proper set. The interior points of $G$ and $G^{\prime}$ form identical sets; it is also readily seen that the points exterior to $G$ and $G^{\prime}$, respectively, also form identical sets, and so the auxiliary boundaries of $G$ and $G^{\prime}$ are identical point sets.

Definition 6. By the partial domain $G$ of $T$ corresponding to the closed partial region $G^{\prime}$ of $T^{\prime}$ we mean the set of all points of $T$ which are interior points of $G^{\prime}$.

Definition 7. An infinite sequence of closed partial regions of $T^{\prime}: G_{1}^{\prime}$, $G_{2}^{\prime}, G_{3}^{\prime}, \cdots$ is said to be monotone if the following conditions are satisfied:

* A boundary point of any given set $E$ is a limit point of points of $E$ which is not interior to $E$; a frontier point of $E$ is a limit point of exterior points of $E$ which is not exterior to $E$. The frontier of a finite domain $T$ is always a proper set and is contained in the boundary, $t$. 
(1) Each point of $G_{i+1}^{\prime}$ is a point of $G_{i}^{\prime}, i=1,2,3, \cdots$.

(2) The auxiliary boundaries of no two of the given closed partial regions have a point in common.

\section{Components}

Definition 8. A component $\Gamma$ of $T^{\prime}$ is a monotone sequence of closed partial regions of $T^{\prime}$, with the convention that two such sequences $G_{1}^{\prime}, G_{2}^{\prime}, G_{3}^{\prime}, \cdots$, and $\bar{G}_{1}^{\prime}, \bar{G}_{2}^{\prime}, \bar{G}_{3}^{\prime}, \cdots$, determine identical components if and only if to every positive integer $i$ there corresponds a pair of positive integers $j$ and $k$ such that $G_{j}^{\prime} \leqq \bar{G}_{i}^{\prime}$ and $\bar{G}_{k}^{\prime} \leqq G_{i}^{\prime}$.

Definition 9. The component $\Gamma: G_{1}^{\prime}, G_{2}^{\prime}, G_{3}^{\prime}, \cdots$ of $T^{\prime}$ is contained in a given finite closed region $E$ (not necessarily a subregion of $T^{\prime}$ ) if and only if there exists a positive integer $i$ such that $G_{i}^{\prime} \leqq E$.

Definition 10. The component $\Gamma: G_{1}^{\prime}, G_{2}^{\prime}, G_{3}^{\prime}, \ldots$ of $T^{\prime}$ is contained in the component $\bar{\Gamma}: \bar{G}_{1}^{\prime}, \bar{G}_{2}^{\prime}, \bar{G}_{3}^{\prime}, \cdots$ of $T^{\prime}$ if and only if $\Gamma$ is contained in each $\bar{G}_{i}^{\prime}$.

We note that two components $\Gamma$ and $\bar{\Gamma}$ are identical if and only if each is contained in the other.

Definition 11. A given point is contained in the component $\Gamma: G_{1}^{\prime}, G_{2}^{\prime}$, $G_{3}^{\prime}, \ldots$ if and only if that point is contained in each $G_{i}^{\prime}$.

TheOrem 2. A component $\Gamma$ of $T^{\prime}$ contains at least one point of $t$.

Let $G_{1}^{\prime}, G_{2}^{\prime}, G_{3}^{\prime}, \cdots$ be a monotone sequence of closed partial regions of $T^{\prime}$ determining $\Gamma$. Now every point of the proper closed point set $t \cdot G_{+1}^{\prime}$ is also a point of the set $t \cdot G_{i}^{\prime}, i=1,2,3, \cdots$. Hence there is at least one point common to the proper closed sets $t \cdot G_{1}^{\prime}, t \cdot G_{2}^{\prime}, t \cdot G_{3}^{\prime}, \ldots$ Such a point belongs to $t$ and is contained in $\Gamma$.

Definition 12. Let $T^{(1)}$ and $T^{(2)}$ be two given finite domains. If there exists an infinite sequence, $G_{1}, G_{2}, G_{3}, \cdots$, of common partial domains of $T^{(1)}$ and $T^{(2)}$ such that the corresponding closed finite regions, $G_{1}^{\prime}, G_{2}^{\prime}, G_{3}^{\prime}, \cdots$, form a monotone sequence of closed partial regions of $T^{(1) \prime}$ and of $T^{(2) '}$, and thus determine components $\Gamma^{(1)}$ of $T^{(1) \prime}$ and $\Gamma^{(2)}$ of $T^{(2) \prime}$, then $\Gamma^{(1)}$ and $\Gamma^{(2)}$ are called equivalent components.

\section{BOUNDARY ELEMENTS}

Definition 13. An element $\gamma$ of the boundary $t$ of $T$ is a component of $T^{\prime}$ which may be determined by a monotone sequence of closed partial regions, $G_{1}^{\prime}, G_{2}^{\prime}, G_{3}^{\prime}, \cdots$, having the property that the diameter* of $G_{i}^{\prime}$ approaches the limit zero as $i$ becomes infinite.

* We adopt the usual definition of the diameter of a closed point set, namely, the maximum distance between two points of the set. 
TheOREM 3. A necessary and sufficient condition that a component $\Gamma$ of $T^{\prime}$ be a boundary element is that $\Gamma$ contain one point of $t$, and no other point.

The necessity of the condition is immediately obvious. To establish the sufficiency we construct a sphere $S$ of arbitrary positive radius, with center at $p$, the single point contained in the component $\Gamma$ determined by the monotone sequence $G_{1}^{\prime}, G_{2}^{\prime}, G_{3}^{\prime}, \cdots$. We denote by $E_{k}$ the set of all points of $G_{k}{ }^{\prime}$ which lie outside or on the boundary of the sphere $S$. Now $E_{k}$ is either a null set or a closed proper set; we note also that $E_{1} \geqq E_{2} \geqq E_{3} \geqq \ldots$. Hence if each $E_{k}$ is a proper set, there is a point $P$, without or on the boundary of $S$, which belongs to each $E_{k}$ and so to each $G_{k}{ }^{\prime}$. But such a point would be a point distinct from $p$ contained in $\Gamma$, contrary to hypothesis. We infer that for some positive integral $k, E_{k}$ is a null set, and that each of the closed partial regions $G_{k}^{\prime}, G_{k+1}^{\prime}, G_{k+2}^{\prime}, \cdots$ contains only points interior to $S$. Since the radius of $S$ is arbitrary, this means that the diameter of $G_{i}^{\prime}$ approaches the limit zero as $i$ becomes infinite, and so $\Gamma$ is a boundary element.

THEOREM 4. If a boundary element $\gamma$ contains a boundary element $\bar{\gamma}$ then $\gamma$ and $\bar{\gamma}$ are identical.

Let $G_{1}^{\prime}, G_{2}^{\prime}, G_{3}^{\prime}, \cdots$ and $\bar{G}_{1}^{\prime}, \bar{G}_{2}^{\prime}, \bar{G}_{3}^{\prime}, \cdots$ be monotone sequences determining the boundary elements $\gamma$ and $\bar{\gamma}$ respectively. We will denote the partial domains of $T$ corresponding to $G_{i}^{\prime}$ and $\bar{G}_{i}^{\prime}$ by $G_{i}$ and $\bar{G}_{i}$ respectively. Clearly, $\gamma$ and $\bar{\gamma}$ contain the same point $p$ of $t$. We may, without loss of generality, assume that $p$ does not lie on the auxiliary boundary of any of the closed partial regions of the second sequence. For, from the definition of a monotone sequence, it could not lie on more than one $\bar{G}_{i}^{\prime}$, and if such an exceptional closed partial region were deleted, we should still have a monotone sequence determining a boundary element identical with $\bar{\gamma}$.

Given an arbitrary $\bar{G}_{i}^{\prime}$, we know that the distance from $p$ to $\bar{g}_{i}$ (the auxiliary boundary of $\left.\bar{G}_{i}^{\prime}\right)$ is positive. Consequently there exists a $G_{j}^{\prime}$ which has no point in common with $\bar{g}_{i}: G_{j}^{\prime} \cdot \bar{g}_{i}=0$. Since $\gamma$ contains $\bar{\gamma}$, it is readily seen that $G_{j}$ contains all points of some $\bar{G}_{k}$ and so some point $Q$ of $\bar{G}_{i}$. Suppose now that there exists a point $Q^{\prime}$ in the interior of $G_{j}$ but exterior to $\bar{G}_{i}$. Then $Q$ and $Q^{\prime}$ can be joined by a continuous curve in $G_{j}$. Such a curve must pass from the interior of $\bar{G}_{i}$ to the exterior of $\bar{G}_{i}$, and so must pass through a point of $\bar{g}_{i}$. But this contradicts the relation $G_{j}^{\prime} \cdot \bar{g}_{i}=0$, and so shows that any point interior to $G_{j}$ is not exterior to $\bar{G}_{i}$, and so is a point of $\bar{G}_{i}^{\prime}$; we infer that given any $\bar{G}_{i}^{\prime}$, there exists a $G_{j}^{\prime}$ such that $G_{j}^{\prime} \leqq \bar{G}_{i}^{\prime}$. This means that $\gamma$ is contained in every closed partial region of a monotone sequence determining $\bar{\gamma}$; that is, $\gamma$ is contained in $\bar{\gamma}$. Since $\gamma$ contains $\bar{\gamma}$ by hypothesis, we conclude that $\gamma$ and $\bar{\gamma}$ are identical. 
Definition 14. Let $P_{1}$ be a point of $T$, and let $\rho$ be any positive quantity. Then $\mathfrak{S}\left(P_{1}, \rho\right)$, the pseudo-spherical domain of radius $\rho$ with center at $P_{1}$, is defined as the finite domain containing each point of $T$ in the interior of the sphere of radius $\rho$ with center at $P_{1}$ which can be joined to $P_{1}$ by a continuous curve made up entirely of such points. The set $\varsigma^{\prime}\left(P_{1}, \rho\right)$ obtained by adjoining to $\mathfrak{S}\left(P_{1}, \rho\right)$ all its limit points, is called the closed pseudo-spherical region of radius $\rho$ with center at $P_{1}$.

The pseudo-spherical domain $\subseteq\left(P_{1}, \rho\right)$ may or may not have an auxiliary boundary. If it has, all points of the auxiliary boundary lie on the surface of the associated sphere. If $\subseteq\left(P_{1}, \rho\right)$ has an auxiliary boundary, then $\varsigma^{\prime}\left(P_{1}, \rho\right)$ has the same auxiliary boundary.

Definition 15. Let $G_{1}^{\prime}, G_{:}^{\prime}, G_{3}^{\prime}, \cdots$ be a monotone sequence of closed partial regions of $T^{\prime}$ determining an element $\gamma$ of $t$ containing the point $p$. Given any positive $\rho$, let $i$ be the smallest integer such that the interior $S$ of the sphere of radius $\rho$ with center at $p$ contains $G_{i}$. Let $P$ be a point of the corresponding $G_{i}$. Then $\subseteq(\gamma, \rho)$, the pseudo-spherical domain of radius $\rho$ corresponding to $\gamma$, is defined as the finite domain consisting of those points which can be joined to $P$ by a continuous curve lying in $T \cdot S$. Also, $\varsigma^{\prime}(\gamma, \rho)$, the closed pseudo-spherical region of radius $\rho$ corresponding to $\gamma$, is defined as the closed finite region obtained by adjoining to $\mathfrak{\subseteq}(\gamma, \rho)$ all its limit points.

THEOREM 5. If, in the definition of $\mathfrak{S}(\gamma, \rho)$ and $\mathfrak{S}^{\prime}(\gamma, \rho)$, the sequence $G_{1}^{\prime}, G_{2}^{\prime}, G_{3}^{\prime}, \ldots$ is replaced by another monotone sequence determining an element of $t$ identical with $\gamma$, the sets $\subseteq(\gamma, \rho)$ and $\varsigma^{\prime}(\gamma, \rho)$ are unaltered; these sets are also independent of the choice of $P$ in $G_{i}$. If $\rho$ is less than $\rho_{0}$, the distance from $p$ to the farthest point or points of $t$, then $\mathfrak{S}(\gamma, \rho)$ is a partial domain of $T$ and $\widetilde{S}^{\prime}(\gamma, \rho)$ is a closed partial region of $T^{\prime}$. Moreover, if $\rho_{0}, \rho_{1}, \rho_{2}, \rho_{3}, \cdots$ is an infinite monotone decreasing sequence of positive numbers tending to zero, then $\Im^{\prime}\left(\gamma, \rho_{1}\right), \mathfrak{S}^{\prime}\left(\gamma, \rho_{2}\right), \mathfrak{S}^{\prime}\left(\gamma, \rho_{3}\right), \cdots$ is a monotone sequence of closed partial regions of $T^{\prime}$ determining an element of $t$ identical with $\gamma$.

Theorem 6. A necessary and sufficient condition that a given point $p$ of $t$ be contained in at least one element of $t$ is that $p$ be an accessible boundary point of $T$.

To establish the sufficiency of the condition we assume that there exists a curve $C$ given parametrically by the equations*

$$
x=X(\theta), \quad y=Y(\theta), \quad z=Z(\theta), \quad 0 \leqq \theta \leqq 1,
$$

\footnotetext{
* It is not necessary to assume that every pair of distinct values of $\theta$ on the interval $0 \leqq \theta \leqq 1$ correspond to distinct points of $C$. However, the definition of accessibility which we use is in reality no more general than that in which this further restriction is imposed on the curve $C$.
} 
where $X(\theta), Y(\theta)$, and $Z(\theta)$ are continuous functions, such that each point of $C$ except that corresponding to $\theta=1$ is in $T$, and the point corresponding to $\theta=1$ is the given point $p$.

Consider the distance from $p$ to the variable point $P$ of $C$. This distance is a continuous function of $\theta$, the parametric coordinate of $P$ on $C$, and assumes a positive maximum value $\rho_{1}$ for a finite or a closed infinite set of values of $\theta$ on the interval $0 \leqq \theta \leqq 1$. In any case there is a largest value of $\theta$, say $\theta=\theta_{1}$, for which this maximum value $\rho_{1}$ is attained. We denote by $G_{1}$ the set containing each point of $T$ which can be joined to the point of $C$ with parametric coordinate $\theta_{1}^{\prime}=\left(1+\theta_{1}\right) / 2$ by a continuous curve lying entirely in $T$ and in the interior of the sphere $S_{1}$ with center at $p$ and radius $\rho_{1}$. We note that all points of that part of $C$ for which $\theta_{1}<\theta<1$ are points of $G_{1}$. All points of the auxiliary boundary of $G_{1}$ are on the surface of the sphere $S_{1}$. The set $G_{1}$ is a partial domain of $T$.

Given any integer $i$ greater than unity, we consider the sphere $S_{i}$ of radius $\rho_{i}=\rho_{1} / 2^{i}$. There exists a constant $\theta_{i}$, less than unity, such that all points of $C$ for which $\theta_{i}<\theta<1$ lie in the interior of $S_{i}$ and the point of $C$ for which $\theta=\theta_{i}$ lies on $S_{i}$. We denote by $G_{i}$ the partial domain* of $T$ which contains each point of $T$ which can be joined by a continuous curve lying in $T$ and in the interior of $S_{i}$ to the point of $C$ with parametric coordinate $\theta_{i}^{\prime}=\left(1+\theta_{i}\right) / 2$.

We now form the sequence of the corresponding closed partial regions of $T^{\prime}: G_{1}^{\prime}, G_{2}^{\prime}, G_{3}^{\prime}, \ldots$ This sequence is monotone, and determines a boundary element containing the given point $p$. This establishes the sufficiency of the given condition.

To prove that the condition is necessary, we assume that $p$ is contained in an element $\gamma$ of $t$ and define a continuous curve approaching $p$ from the interior of $T$. Let $\rho$ be a positive constant less than the distance from $p$ to the farthest point or points of $t$, and let $P$ be an arbitrary point of the pseudospherical domain $\subseteq(\gamma, \rho)$. The distance from $P$ to the boundary of $\subseteq(\gamma, \rho)$ attains its maximum value on a finite or closed infinite subset $\mathfrak{S}_{1}$ of points in $\mathfrak{S}(\gamma, \rho)$. In either case it is possible to give a law whereby we may select uniquely $\dagger$ (relatively to an arbitrarily preassigned Cartesian coordinate sys-

* It may be noted that the determination of $G_{i}$ depends on $C$, but is independent of the parametric representation of this curve, provided the point $p$ corresponds to $\theta=1$ and the other end of $C$ to $\theta=0$.

$\dagger$ For instance, we may discard all points of the set $\aleph_{1}$ except those for which the $x$-coordinate attains its maximum value. If this does not restrict us to a single point, we may then discard from the remaining points all except those on which the $y$-coordinate attains its maximum value. If more than one point remains, we may choose as $Q_{1}$ the unique point on which the $z$-coordinate attains its maximum value. This method may be used to select uniquely a point from any bounded closed set. The purpose of prescribing a law for the choice of $Q_{1}$ is to show that it is not necessary to make an infinite number of arbitrary choices to obtain the set of points $Q_{i}, i=1,2,3, \cdots$. 
tem) a particular point $Q_{1}$ from $\mathfrak{S}_{1}$. There exists a largest integer $k_{1}$ such that $Q_{1}$ is a point of $\subseteq\left(\gamma, 2^{-k_{1}} \rho\right)$. We now determine uniquely a particular point $Q_{2}$ of $\mathfrak{S}\left(\gamma, 2^{-\left(k_{1}+1\right)} \rho\right)$ by the method that we used to select $Q_{1}$ from $\mathfrak{S}(\gamma, \rho)$. There exists a largest integer $k_{2}$ such that $Q_{2}$ is a point of $\subseteq\left(\gamma, 2^{-k_{2}} \rho\right)$. We note that $k_{2}>k_{1}$. By repetitions of this process we obtain an infinite sequence of points $Q_{1}, Q_{2}, Q_{3}, \cdots$, and an infinite sequence of integers

$$
k_{1}<k_{2}<k_{3}<\cdots \text {, }
$$

such that $Q_{i}$ is a point of $\subseteq\left(\gamma, 2^{-k_{i}} \rho\right)$ but not a point of $\subseteq\left(\gamma, 2^{-k_{i+1}} \rho\right)$, where $i=1,2,3, \cdots$.

We now show that it is possible to state a law whereby, given any positive integer $i$, we may determine uniquely a broken line of a finite number of segments lying entirely in $\mathfrak{S}\left(\gamma, 2^{-k_{i}} \rho\right)$ and joining $Q_{i}$ and $Q_{i+1}$. There exists a smallest integer $m_{i}$ such that $Q_{i}$ and $Q_{i+1}$ can be joined by broken line of $m_{i}$ segments lying entirely in $\mathfrak{S}\left(\gamma, 2^{-k_{i}} \rho\right)$. If $m_{i}>1$ the set of all points $Q$ such that $\overline{Q_{i} Q}$ is a segment of such a broken line of $m_{i}$ segments is a proper open subset of $\mathfrak{S}\left(\gamma, 2^{-k_{i}} \rho\right)$. We may select uniquely a particular point $Q_{i, 1}$ from this subset by restricting ourselves first to those points at a maximum distance from the boundary of the subset, and then using the device employed to choose the point $Q_{1}$ from $\mathfrak{S}_{1}$. Now $Q_{i, 1}$ can be joined to $Q_{i+1}$ by a broken line of $m_{i}-1$ segments, lying entirely in $\subseteq\left(\gamma, 2^{-k_{i}} \rho\right)$. Using the method employed above, we may select uniquely a point $Q_{i, 2}$ in such a way that $Q_{i, 1} Q_{i, 2}$ may be used as a segment of this broken line. By successive repetitions of this process we determine uniquely a broken line $Q_{i}, Q_{i, 1}, Q_{i, 2}, \cdots$, $Q_{i, m_{i}-1}, Q_{i+1}$, lying entirely in $\Im\left(\gamma, 2^{-k_{i}} \rho\right)$ and joining $Q_{i}$ and $Q_{i+1}$.

By applying this procedure to each pair of successive points in the sequence $Q_{1}, Q_{2}, Q_{3}, \cdots$, and adjoining to the set of all points on all the line segments the point $p$, we obtain a continuous curve terminating at $p$, but otherwise lying entirely in $T$. It is a simple matter to establish a parametric representation for the curve, if desired. This shows that $p$ is an accessible boundary point of $T$ and completes the proof of the theorem.

Corollary. Given a point $P$ of $T$ at a distance $\bar{\rho}$ from $t$, there exists at least one element $\gamma$ of $t$ such that $P$ is a point of every pseudo-spherical domain $\subseteq(\gamma, \rho)$ such that $\rho>\bar{\rho}$. Moreover, it is possible to prescribe a law whereby such an element $\gamma$ of $t$ is determined uniquely by a given point $P$ of $T$ and a given Cartesian coordinate system.

Let $p$ be a point of $t$ on which the distance from $P$ to a variable point of $t$ attains its minimum value, $\bar{\rho}$. The set of points on $t$ satisfying this requirement form a proper finite or a closed infinite set, and a unique choice may 
be made by the law described in connection with a similar situation in the discussion of Theorem 6. From the discussion of this theorem, we know that there exists a unique element $\gamma$ of $t$ determined by the line segment $C$ joining $P$ and $p$. Clearly $P$ is a point of $\subseteq(\gamma, \rho)$, provided $\rho>\bar{\rho}$.

\section{Functions of Boundary ELEMENTS}

\section{Pseudo-continuity}

Definition 16. A function of a variable element of the boundary $t$ of $T$ is defined when a law* is given whereby to each element $\gamma$ of $t$ there corresponds a uniquely determined real number, $f(\gamma)$.

Definition 17. A function $f(\gamma)$ of a variable element $\gamma$ of $t$ is said to be pseudo-continuous at the element $\gamma=\gamma_{1}$, if to each positive quantity $\epsilon$ there corresponds a positive quantity $\delta$ such that

$$
\left|f(\gamma)-f\left(\gamma_{1}\right)\right|<\epsilon
$$

for all elements $\gamma$ of $t$ contained in the closed pseudo-spherical region $\varsigma^{\prime}\left(\gamma_{1}, \delta\right)$.

Definition 18. A function $f(\gamma)$ of a variable element $\gamma$ of $t$ is pseudo-continuous on $t$ if it is pseudo-continuous at each element of $t$.

Definition 19. A function $f(\gamma)$ of a variable element $\gamma$ of $t$ is said to be uniformly pseudo-continuous on $t$ if, given $\gamma_{1}$ and an arbitrary positive quantity $\epsilon$, there exists a positive quantity $\delta$, independent of $\gamma_{1}$, such that

$$
\left|f(\gamma)-f\left(\gamma_{1}\right)\right|<\epsilon,
$$

for all elements $\gamma$ of $t$ contained in the closed pseudo-spherical region $\varsigma^{\prime}\left(\gamma_{1}, \delta\right)$.

To any given function $\phi(p)$ of a variable point $p$ of $t$ there corresponds a function $\dagger f(\gamma)$ of the variable element $\gamma$ of $t$ obtained by assigning to an arbitrary element $\gamma$ of $t$ the value assumed by the function $\phi(p)$ at the point $p$ contained in $\gamma$. If the function $\phi(p)$ is continuous at a particular point $p_{1}$ of $t$,

* The definition of a function of a variable element of $t$ is analogous to Dirichlet's well known definition of a function of a real variable. An alternative form of the definition of the new concept is given below. Let $\mathfrak{F}[X(\theta), Y(\theta), Z(\theta)]$ be any functional of the real functions $X(\theta), Y(\theta), Z(\theta)$, $0 \leqq \theta \leqq 1$, which (1) is defined, single-valued and real for each choice of this triple of functions determining a continuous curve $C: x=X(\theta), y=Y(\theta), z=Z(\theta)$ having the end $\theta=1$ on $t$ but otherwise lying in $T$, and which (2) has the property that if any two triples of such functions $X_{1}(\theta), Y_{1}(\theta), Z_{1}(\theta)$ and $X_{2}(\theta), Y_{2}(\theta), Z_{2}(\theta)$ determine curves yielding (by application of the process described in the first part of the proof of Theorem 6) identical elements of $t$, then

$$
\mathfrak{F}\left[X_{1}(\theta), Y_{1}(\theta), Z_{1}(\theta)\right]=\mathfrak{F}\left[X_{2}(\theta), Y_{2}(\theta), Z_{2}(\theta)\right] \text {. }
$$

Any such functional $\mathfrak{F}[X(\theta), Y(\theta), Z(\theta)]$ gives rise to a function $f(\gamma)$ of the variable element $\gamma$ of $t$ which assumes at a given element $\gamma$ of $t$ the value of the functional for functions $X(\theta), Y(\theta)$ and $Z(\theta)$ determining any curve yielding the boundary element $\gamma$.

$\dagger$ This is, of course, a very special type of function of $\gamma$. 
then the corresponding function $f(\gamma)$ is pseudo-continuous at each element which contains $p_{1}$. If $\phi(p)$ is continuous at each point of $t$, and therefore uniformly continuous on $t$, then $f(\gamma)$ is uniformly pseudo-continuous on $t$.

We note, however, that even though $\phi(p)$ be discontinuous on $t$, in fact unbounded on $t$, the function $f(\gamma)$ may nevertheless be uniformly pseudocontinuous on $t$. Consider, for instance, the case in which $T$ is the domain bounded by the surfaces

$$
\begin{aligned}
x^{2}+y^{2} & =9, & & 0<z<1 ; \\
z & =0, & & x^{2}+y^{2} \leqq 9 ; \\
z & =1, & & x^{2}+y^{2} \leqq 9 ; \\
z & =(2 i)^{-1 / 2}, & & x^{2}+y^{2} \leqq 9, \quad(x-2)^{2}+y^{2} \geqq \frac{1}{4}, \quad i=1,2,3, \cdots, \\
z & =(2 i+1)^{-1 / 2}, & & x^{2}+y^{2} \leqq 9, \quad(x+2)^{2}+y^{2} \geqq \frac{1}{4}, \quad i=1,2,3, \cdots .
\end{aligned}
$$

We define $\phi(p)$ at the boundary point $p:(x, y, z)$ so that $\phi(p)=z^{-1}$ when $z \neq 0$ and $\phi(p)=1$ when $z=0$.

A boundary point for which $z=0$ is not contained in any boundary element. Given a boundary element $\gamma_{1}$ of $t$ containing a point $p_{1}:\left(x_{1}, y_{1}, z_{1}\right)$ such that $z_{1} \leqq \frac{1}{2}$, there exists an integer $m_{1}>4$ such that $m_{1}^{-1 / 2}<z_{1} \leqq\left(m_{1}-1\right)^{-1 / 2}$. For each point $p:(x, y, z)$ contained in an element $\gamma$ contained in $\widetilde{S}^{\prime}\left(\gamma_{1}, 1\right)$ we have $\left(m_{1}+2\right)^{-1 / 2} \leqq z \leqq\left(m_{1}-3\right)^{-1 / 2}$, and so

$$
\left(m_{1}-3\right)^{1 / 2}-m_{1}^{1 / 2}<z^{-1}-z_{1}^{-1} \leqq\left(m_{1}+2\right)^{1 / 2}-\left(m_{1}-1\right)^{1 / 2},
$$

whence,

$$
\left|f(\gamma)-f\left(\gamma_{1}\right)\right|<3 m_{1}^{-1 / 2}<3 z_{1} .
$$

Given any positive $\epsilon$, we infer that if $\gamma_{1}$ is so chosen that $z_{1} \leqq \epsilon / 3$ and $z_{1} \leqq \frac{1}{2}$, then throughout $\widetilde{S}^{\prime}\left(\gamma_{1}, 1\right)$ we have

$$
\left|f(\gamma)-f\left(\gamma_{1}\right)\right|<\epsilon .
$$

Let $\bar{t}$ be the subset of $t$ each point of which is contained in a pseudospherical region of unit radius corresponding to some boundary element containing a point on or above at least one of the planes $z=\epsilon / 3$ and $z=\frac{1}{2}$. To the given $\epsilon$ there corresponds a positive quantity $\bar{\delta}$, independent of the choice of the point $\bar{p}:(\bar{x}, \bar{y}, \bar{z})$ of $\bar{t}$, such that for each point $p:(x, y, z)$ of $t$ at a distance from $\bar{p}$ less than $\bar{\delta}$ we have

$$
|\phi(p)-\phi(\bar{p})|<\epsilon .
$$

If, now, we choose $\delta$ as the smaller of the two numbers $\bar{\delta}$ and unity, or their common value if $\bar{\delta}=1$, then, given any element $\gamma_{2}$ of $t$ and any element $\gamma$ of $t$ contained in $\widetilde{S}^{\prime}\left(\gamma_{2}, \delta\right)$, we may write 


$$
\left|f(\gamma)-f\left(\gamma_{2}\right)\right|<\epsilon .
$$

Hence the function $f(\gamma)$ is uniformly pseudo-continuous on $t$, even though it has no upper bound on $t$. It is a simple matter to extend the method used in the construction of this example so as to obtain a function with neither an upper nor a lower bound on the boundary of a certain finite domain, but which is nevertheless uniformly pseudo-continuous on the boundary of that domain.

Definition 20. A function $F(P)$ of the variable point $P$ of $T$ is said to approach a given value $c$ at a given boundary element $\gamma$ of $t$ if to each positive quantity $\epsilon$ there corresponds a positive quantity $\delta$ such that

$$
|F(P) \rightarrow c|<\epsilon,
$$

throughout the pseudo-spherical domain $\subseteq(\gamma, \delta)$.

Definition 21. Given a function $F(P)$ of a variable point $P$ of $T$, and a function $f(\gamma)$ of a variable element $\gamma$ of $t$, the function $F(P)$ is said to approach the boundary values $f(\gamma)$ with uniform pseudo-continuity if, given an arbitrary positive quantity $\epsilon$, there exists a positive quantity $\delta$, independent of $\gamma$, such that

$$
|F(P)-f(\gamma)|<\epsilon,
$$

throughout the pseudo-spherical domain $\subseteq(\gamma, \delta)$.

Definition 22. A function $F(P)$ of a variable point $P$ of $T$ is said to be pseudo-uniformly continuous in $T$ if, given an arbitrary positive quantity $\epsilon$, there exists a positive quantity $\delta$, independent of $P_{1}$, such that

$$
\left|F(P)-F\left(P_{1}\right)\right|<\epsilon,
$$

for all points $P$ of the pseudo-spherical domain $\subseteq\left(P_{1}, \delta\right)$.

A slightly different formulation of the property here involved is useful. It is embodied in the following proposition:

TheOREM 7. A necessary and sufficient condition that a function $F(P)$ be pseudo-uniformly continuous in $T$ is that given any positive quantity $\epsilon$, there exists a positive quantity $\delta$ such that

$$
\left|F(P)-F\left(P^{\prime}\right)\right|<\epsilon,
$$

for every pair of points $P$ and $P^{\prime}$ in $T$ that can be joined by a continuous curve (lying entirely in $T$ ) the maximum distance between two points of which is less than $\delta$.

Let $F(P)$ be a function of the point $P$ of $T$. If to a given positive $\epsilon$ there corresponds a $\delta$ for which the condition given in Definition 22 holds, then 
the condition given in this theorem also holds for the same $\epsilon$ and $\delta$. If to a given positive $\epsilon$ there corresponds a $\delta$ such that the condition given in this theorem holds, the condition given in Definition 22 holds for the given $\epsilon$ provided $\subseteq\left(P_{1}, \delta\right)$ is replaced by $\subseteq\left(P_{1}, \delta / 2\right)$.

THEOREM 8. A necessary and sufficient condition that a function $F(P)$ of a variable point $P$ of $T$ be continuous in $T$ and approach bounded and uniformly pseudo-continuous boundary values on $t$ with uniform pseudo-continuity is that $F(P)$ be bounded and pseudo-uniformly continuous in $T$.

If $F(P)$ is continuous in $T$ and approaches with uniform pseudo-continuity the bounded and uniformly pseudo-continuous boundary values $f(\gamma)$ on $t$, then corresponding to any given positive $\epsilon$ there exists a positive $\delta_{1}$, independent of the arbitrary element $\gamma$ of $t$, such that

$$
|F(P)-f(\gamma)|<\frac{\epsilon}{2}
$$

throughout $\Im\left(\gamma, 3 \delta_{1}\right)$. Now the function $F(P)$ is bounded and uniformly continuous on the closed set comprising points of $T$ at a distance at least $\delta_{1}$ from $t$; hence to the given $\epsilon$ there corresponds a positive $\delta_{2}$ such that

$$
\left|F\left(P_{1}\right)-F\left(P_{2}\right)\right|<\epsilon,
$$

for all pairs of points $P_{1}$ and $P_{2}$ of this subset of $T$ which are so located that $\overline{P_{1} P_{2}}<\delta_{2}$. From these relations and the Corollary of Theorem 6, it follows that if $\delta$ is the smaller of $\delta_{1}$ and $\delta_{2}$ (or their common value if they are equal) then

$$
\left|F(P)-F\left(P^{\prime}\right)\right|<\epsilon,
$$

for all points $P$ in $\Im\left(P^{\prime}, \delta\right)$, where $P^{\prime}$ is an arbitrary point of $T$. Hence the given condition is necessary.

Assuming now that $F(P)$ is bounded and pseudo-uniformly continuous in $T$, let $\gamma$ be an arbitrary element of $t$, and $\rho$ an arbitrary positive quantity. We denote by $H(\gamma, \rho)$ and $h(\gamma, \rho)$ the least upper and greatest lower bounds, respectively, of $F(P)$ in $\subseteq(\gamma, \rho)$. We note that as $\rho$ decreases in value, $H(\gamma, \rho)$ never increases and $h(\gamma, \rho)$ never decreases. Consequently, for any fixed element $\gamma, \lim _{\rho \rightarrow 0} H(\gamma, \rho)$ and $\lim _{\rho \rightarrow 0} h(\gamma, \rho)$ exist, and

$$
h(\gamma, \rho) \leqq \lim _{\rho \rightarrow 0} h(\gamma, \rho) \leqq \lim _{\rho \rightarrow 0} H(\gamma, \rho) \leqq H(\gamma, \rho) .
$$

Theorem 7 implies that, given any positive $\epsilon$, there exists a positive $\delta$, independent of $\gamma$, such that 


$$
0 \leqq H(\gamma, \delta)-h(\gamma, \delta)<\epsilon .
$$

Hence,

$$
\lim _{\rho \rightarrow 0} H(\gamma, \rho)=\lim _{\rho \rightarrow 0} h(\gamma, \rho)
$$

The common value of these limits is a bounded function of $\gamma$ on $t$; we represent this function by $f(\gamma)$.

Using Theorem 7 it is easy to show that, given any positive $\epsilon$, there exists a positive $\delta$ independent of $P, P^{\prime}$ and the arbitrary element $\gamma_{1}$ of $t$, such that

$$
\left|F(P)-F\left(P^{\prime}\right)\right|<\frac{\epsilon}{3}
$$

for every pair of points $P$ and $P^{\prime}$ in $\subseteq\left(\gamma_{1}, \delta\right)$. If $0<\rho<\delta$, we have then, in $\Im\left(\gamma_{1}, \rho\right)$,

whence,

$$
\left|F(P)-H\left(\gamma_{1}, \rho\right)\right| \leqq \frac{\epsilon}{3}
$$

$$
\left|F(P)-f\left(\gamma_{1}\right)\right| \leqq \frac{\epsilon}{3}<\epsilon
$$

which proves that $F(P)$ approaches the boundary values $f(\gamma)$ with uniform pseudo-continuity. Moreover, if $\gamma$ is an element of $t$ contained in $\mathfrak{S}^{\prime}\left(\gamma_{1}, \delta\right)$ there exists a positive $\rho^{\prime}$ so small that $\mathfrak{S}\left(\gamma, \rho^{\prime}\right)$ is a subset of $\mathfrak{S}\left(\gamma_{1}, \delta\right)$; hence the relations

$$
\left|F(P)-F\left(P^{\prime}\right)\right|<\frac{\epsilon}{3}, \quad\left|F(P)-f\left(\gamma_{1}\right)\right| \leqq \frac{\epsilon}{3}, \text { and }\left|F\left(P^{\prime}\right)-f(\gamma)\right| \leqq \frac{\epsilon}{3}
$$

are valid for $P$ in $\subseteq\left(\gamma_{1}, \delta\right)$ and $P^{\prime}$ in $\subseteq\left(\gamma, \rho^{\prime}\right)$ and imply that

$$
\left|f(\gamma)-f\left(\gamma_{1}\right)\right|<\epsilon .
$$

This shows that $f(\gamma)$ is uniformly pseudo-continuous on $t$, and so completes the proof of the theorem.

THEOREM 9. Given any bounded and uniformly pseudo-continuous function $f(\gamma)$ of a variable element $\gamma$ of $t$, there exists a function $F(P)$ of the variable point $P$ of $T$ which is bounded and continuous in $T$ and approaches the boundary values $f(\gamma)$ with uniform pseudo-continuity.

This proposition corresponds to part of a theorem regarding functions 
continuous on the boundary of a domain established by Lebesgue.* The proof given below is an adaptation of a proof of Lebesgue's theorem due to Carathéodory. $\dagger$

Let $P$ be any point of $T$. Let $\rho(P)$ be the distance from $P$ to $t$. We define $u_{P}(r)$ for $r>\rho(P)$ as the least upper bound of $f(\gamma)$ for all elements of $t$ contained in $\varsigma^{\prime}(P, r)$; as $r$ increases $u_{P}(r)$ never decreases, and we may define a function $F(P)$ in $T$ by the formula

$$
F(P)=\frac{1}{\rho(P)} \int_{\rho(P)}^{2 \rho(P)} u_{P}(r) d r .
$$

This function has the desired properties, as is proved below.

Given any positive $\epsilon$ there exists a positive $\delta$, independent of the arbitrarily chosen element $\gamma_{1}$ of $t$, such that

$$
\left|f(\gamma)-f\left(\gamma_{1}\right)\right|<\frac{\epsilon}{2}
$$

for each $\gamma$ contained in $\Im^{\prime}\left(\gamma_{1}, \delta\right)$. If we choose $P$ in $\subseteq\left(\gamma_{1}, \delta / 3\right)$ and restrict $r$ by the inequality $\rho(P)<r<2 \rho(P)$, then $\varsigma^{\prime}(P, r)$ is a subset of $\mathfrak{S}^{\prime}\left(\gamma_{1}, \delta\right)$ and consequently

$$
\left|u_{P}(r)-f\left(\gamma_{1}\right)\right| \leqq \frac{\epsilon}{2}<\epsilon
$$

Accordingly,

$$
\left|F(P)-f\left(\gamma_{1}\right)\right| \leqq \frac{1}{\rho(P)} \int_{\rho(P)}^{2 \rho(P)} \frac{\epsilon}{2} d r<\epsilon,
$$

for any $P$ in $\subseteq\left(\gamma_{1}, \delta / 3\right)$. Hence $F(P)$ approaches the boundary values $f(\gamma)$ with pseudo-uniform continuity.

The function $F(P)$ is clearly bounded in $T$. Since $\rho(P)$ is plainly a nonvanishing continuous function of $P$ in $T$, we may infer the continuity of $F(P)$ in $T$ if we can show that the function

$$
\bar{F}(P)=\int_{\rho(P)}^{2 \rho(P)} u_{P}(r) d r
$$

is continuous in $T$. Let $P$ and $P^{\prime}$ be any two points of $T$ such that $\overline{P P}^{\prime}$ is less than each of the quantities $\rho(P), \rho\left(P^{\prime}\right)$ and $m^{-1} \epsilon / 6$ where $m$ is the least upper bound of $|f(\gamma)|$ on $t$ and $\epsilon$ is an arbitrarily chosen positive constant. Since

* H. Lebesgue, Sur le problème de Dirichlet, Rendiconti del Circolo Matematico di Palermo, vol. 24 (1907), pp. 371-402. See particularly pp. 379, 380.

† C. Carathéodory, Vorlesungen über reelle Funktionen, 2d edition, Leipzig, 1918, pp. 617-618. 
the value of $u_{P}(r)$ for any $r$ such that $\rho(P)<r<2 \rho(P)$ depends on the values of $f(\gamma)$ at elements $\gamma$ of $t$ contained in $\mathfrak{S}^{\prime}(P, r)$ which is a subset of $\mathfrak{S}^{\prime}\left(P^{\prime}\right.$, $\left.r+\overline{P P}^{\prime}\right)$, we see that

$$
\begin{aligned}
\left|\bar{F}(P)-\bar{F}\left(P^{\prime}\right)\right| & \leqq\left|\int_{\rho(P)}^{2 \rho(P)} u_{P^{\prime}}\left(r+\overline{P P^{\prime}}\right) d r-\int_{\rho\left(P^{\prime}\right)}^{2 \rho\left(P^{\prime}\right)} u_{P^{\prime}}(r) d r\right| \\
& =\left|\int_{\rho(P)+\overline{P P^{\prime}}}^{2 \rho(P)+2 \overline{P P^{\prime}}} u_{P^{\prime}}(r) d r-\int_{\rho\left(P^{\prime}\right)}^{2 \rho\left(P^{\prime}\right)} u_{P^{\prime}}(r) d r\right|,
\end{aligned}
$$

whence,

$$
\begin{aligned}
\left|\bar{F}(P)-\bar{F}\left(P^{\prime}\right)\right| & \leqq \int_{\rho(P)+\overline{P P^{\prime}}}^{\rho\left(P^{\prime}\right)}\left|u_{P^{\prime}}(r)\right| d r+\int_{2 \rho\left(P^{\prime}\right)}^{2 \rho(P)+2 \overline{P P^{\prime}}}\left|u_{P^{\prime}}(r)\right| d r \\
& \leqq 6 m \overline{P P^{\prime}}<\epsilon .
\end{aligned}
$$

Hence $F(P)$ is continuous in $T$, and the proof is complete.

THEOREM 10. Let $F(P)$ be a function of the variable point $P$ of $T, \phi(p)$ a continuous function of the variable point $p$ of $t$, and $f(\gamma)$ the function of the variable element $\gamma$ of $t$ which at each element $\gamma$ has the same value as does $\phi(p)$ at the point $p$ contained in $\gamma$. Then a necessary and sufficient condition that $F(P)$ approach the boundary values $f(\gamma)$ with uniform pseudo-continuity is that $F(P)$ approach the boundary values $\phi(p)$ continuously.

The sufficiency of the condition is immediately obvious. The necessity is readily established by observing that the definition of the function $f(\gamma)$ implies that $f(\gamma)$ is uniformly pseudo-continuous on $t$, and by using the first part of the Corollary of Theorem 6.

COROLLARY. Given any uniformly pseudo-continuous function $f(\gamma)$ of a variable element of $t$, and two functions, $F_{1}(P)$ and $F_{2}(P)$ of a variable point $P$ of $T$, each of which approaches the boundary values $f(\gamma)$ with uniform pseudo-continuity, then the function $F(P)=F_{1}(P)-F_{2}(P)$ approaches zero continuously on $t$.

\section{AN APPROXIMATION THEOREM}

The theorem to which this section is devoted does not itself directly involve functions of boundary elements, but is essential for later work.

THEOREM 11. Given an arbitrary Cartesian coordinate system, a positive constant $\epsilon$, and a function $F(P)$ (of the variable point $P:(x, y, z)$ of $T$ ) bounded and pseudo-uniformly continuous in $T$, then there exists a function $F(P)$, defined in $T$, which has the following properties:

(1) The function $\mathfrak{F}(P)$ is bounded and pseudo-uniformly continuous in $T$. 
(2) The function $\mathcal{F}(P)$ has continuous partial derivatives of the first and second order in $T$, and a bounded Laplacian in $T$.

(3) Throughout $T$,

$$
|\mathscr{F}(P)-F(P)|<\epsilon .
$$

Since $F(P)$ is bounded and pseudo-uniformly continuous in $T$, we know that corresponding to the given positive $\epsilon$ there exists a positive $\delta$ such that the relation

$$
|F(P)-F(Q)|<\frac{\epsilon}{2}
$$

holds for every pair of points $P$ and $Q$ in $T$ that can be joined by a continuous curve (lying entirely in $T$ ) the maximum distance between two points of which is less than $10 \delta$. We also know that there exists a positive constant $M$ such that $|F(P)| \leqq M$ throughout $T$.

We shall find it convenient to represent by $\mathfrak{D}(\Pi, \rho ; P)$ the finite domain containing each point of $T$ which can be joined to the point $P:(x, y, z)$ of $T$ by a continuous curve lying in $T$ and in the interior of the sphere of radius $\rho$ with center at a point $\Pi:(\xi, \eta, \zeta)$ whose distance from $P$ is less than $\rho$. It is not required that $\Pi$ be a point of $T$. Every boundary point of $\mathfrak{D}(\Pi, \rho ; P)$ is either a point of $t$ or a boundary point of the sphere described above.

We now define a function $F_{0}(\Pi, P)$, for any point $P$ of $T$ and any point $\Pi$ whose distance from $P$ is less than $5 \delta$, as the greatest lower bound of $F(Q)$ for all points $Q$ in $\mathfrak{D}(\Pi, 5 \delta ; P)$. Clearly,

$$
\left|F_{0}(\Pi, P)\right| \leqq M
$$

for all admissible pairs of points, $\Pi$ and $P$.

We consider now the behavior of $F_{0}\left(\Pi, P^{\prime}\right)$, where $P^{\prime}$ is an arbitrarily chosen fixed point of $T$ and $\Pi$ a variable point in the interior of the sphere $S_{4}$ of radius $4 \delta$ with center at $P^{\prime}$. Given any positive $\epsilon_{1}$ there exists a continuous curve $C$ lying in $\mathfrak{D}\left(\Pi, 5 \delta ; P^{\prime}\right)$ and joining $P^{\prime}$ to a point $\bar{P}$ such that

$$
F(\bar{P})<F_{0}\left(\Pi, P^{\prime}\right)+\epsilon_{1} .
$$

Let $\delta_{1}$ be the distance from $C$ to the surface of the sphere of radius $5 \delta$ with center at $\Pi$. Let $\Pi^{\prime}$ be any point whose distance from $P^{\prime}$ is less than $4 \delta$ and whose distance from $\Pi$ is less than $\delta_{1}$. Then $\bar{P}$ is a point of $\mathfrak{D}\left(\Pi^{\prime}, 5 \delta ; P^{\prime}\right)$, and

$$
F_{0}\left(\Pi^{\prime}, P^{\prime}\right) \leqq F(\bar{P})<F_{0}\left(\Pi, P^{\prime}\right)+\epsilon_{1} .
$$

This relation shows that for fixed $P^{\prime}$ in $T, F_{0}\left(\Pi, P^{\prime}\right)$ is an upper semi-continuous function of $\Pi$ within the sphere $S_{4}$. 
We now define the function $F_{1}\left(\Pi_{1}, P\right)$ for certain pairs of points by requiring that at each point $\Pi_{1}:\left(\xi_{1}, \eta_{1}, \zeta_{1}\right)$ (not necessarily in $T$ ) whose distance from the arbitrary point $P:(x, y, z)$ of $T$ is less than $3 \delta$ we have

$$
F_{1}\left(\Pi_{1}, P\right)=\frac{1}{\delta^{3}} \int_{\zeta_{1}-\delta / 2}^{\zeta_{1}+\delta / 2} d \zeta \int_{\eta_{1}-\delta / 2}^{\eta_{1}+\delta / 2} d \eta \int_{\xi_{1}-\delta / 2}^{\xi_{1}+\delta / 2} F_{0}(\Pi, P) d \xi .
$$

In the integral above, the point $P:(x, y, z)$ is held fixed in $T$, and the integration is performed with respect to the coordinates $\xi, \eta, \zeta$ of the variable point II over the closed cube

$\Re_{1}^{\prime}: \xi_{1}-\frac{\delta}{2} \leqq \xi \leqq \xi_{1}+\frac{\delta}{2}, \eta_{1}-\frac{\delta}{2} \leqq \eta \leqq \eta_{1}+\frac{\delta}{2}, \zeta_{1}-\frac{\delta}{2} \leqq \zeta \leqq \zeta_{1}+\frac{\delta}{2}$.

Every point of this closed cube is within the open sphere of radius $4 \delta$ with center at $P$, and so we know, from properties of the integrand derived above, that the integral has a meaning. Moreover $\left|F_{1}\left(\Pi_{1}, P\right)\right| \leqq M$, for all admissible pairs of points $\Pi_{1}$ and $P$.

We shall establish now an inequality which will prove useful later. For all pairs of points $\Pi_{1}$ and $P$ for which $F_{1}\left(\Pi_{1}, P\right)$ is defined, we have

For

$$
\left|F_{1}\left(\Pi_{1}, P\right)-F(P)\right| \leqq \frac{\epsilon}{2} .
$$

$$
\left|F_{1}\left(\Pi_{1}, P\right)-F(P)\right| \leqq \frac{1}{\delta^{3}} \int_{\zeta_{1}-\delta / 2}^{\zeta_{1}+\delta / 2} d \zeta \int_{\eta_{1}-\delta / 2}^{\eta_{1}+\delta / 2} d \eta \int_{\xi_{1}-\delta / 2}^{\xi_{1}+\delta / 2}\left|F_{0}(\Pi, P)-F(P)\right| d \xi,
$$

and it is easily seen that, for all points $\Pi$ in the region of integration,

$$
\left|F_{0}(\Pi, P)-F(P)\right| \leqq \frac{\epsilon}{2},
$$

whence,

$$
\left|F_{1}\left(\Pi_{1}, P\right)-F(P)\right| \leqq \frac{\epsilon}{2} .
$$

If $P$ is any fixed point of $T$, then $F_{1}\left(\Pi_{1}, P\right)$ is a continuous function of $\Pi_{1}$, throughout the interior of the sphere of radius $3 \delta$ with center at $P$. We may therefore define a function $\mathcal{F}(P)$, for an arbitrary point $P:(x, y, z)$ of $T$, by setting

$$
\begin{aligned}
& \mathscr{F}(P)= \\
& \frac{1}{\delta^{6}} \int_{z-\delta / 2}^{z+\delta / 2} d \zeta_{2} \int_{y-\delta / 2}^{y+\delta / 2} d \eta_{2} \int_{x-\delta / 2}^{x+\delta / 2} d \xi_{2} \int_{\zeta_{2}-\delta / 2}^{\zeta_{2}+\delta / 2} d \zeta_{1} \int_{\eta_{2}-\delta / 2}^{\eta_{2}+\delta / 2} d \eta_{1} \int_{\xi_{2}-\delta / 2}^{\xi_{2}+\delta / 2} F_{1}\left(\Pi_{1}, P\right) d \xi_{1} .
\end{aligned}
$$


For if $P$ is a given fixed point of $T$, the existence of $F(P)$ depends only on the behavior of the integrand, $F_{1}\left(\Pi_{1}, P\right)$, for points $\Pi_{1}$ in the closed cube

$\Re_{2}{ }^{\prime}: \quad x-\delta \leqq \xi_{1} \leqq x+\delta, \quad y-\delta \leqq \eta_{1} \leqq y+\delta, \quad z-\delta \leqq \zeta_{1} \leqq z+\delta$,

no point of which is at a distance from $P$ greater than $3^{1 / 2} \delta$. The function $F_{1}\left(\Pi_{1}, P\right)$ is continuous throughout the range of integration.

For any point $P$ of $T$, we have $|\mathcal{F}(P)| \leqq M$, and also

$$
\begin{aligned}
\mid \mathcal{F}(P) & -F(P) \mid \\
& \leqq \frac{1}{\delta^{6}} \int_{z-\delta / 2}^{z+\delta / 2} d \zeta_{2} \int_{y-\delta / 2}^{y+\delta / 2} d \eta_{2} \int_{x-\delta / 2}^{x+\delta / 2} d \xi_{2} \int_{\zeta_{2}-\delta / 2}^{\zeta_{2}+\delta / 2} d \zeta_{1} \int_{\eta_{2}-\delta / 2}^{\eta_{2}+\delta / 2} d \eta_{1} \int_{\xi_{2}-\delta / 2}^{\xi_{2}+\delta / 2} \\
& \left|F_{1}\left(\Pi_{1}, P\right)-F(P)\right| d \xi_{1},
\end{aligned}
$$

from which we obtain

$$
|\mathfrak{F}(P)-F(P)| \leqq \frac{\epsilon}{2}<\epsilon .
$$

Hence $F(P)$ has the properties given in the first part of item (1) and in item (3) of the conclusion of the theorem.

Let $P^{\prime}$ be any point of $T$, and let $S_{4}$ be the set of all points within the sphere of radius $4 \delta$ with center at $P^{\prime}$. If $\Pi$ is a fixed point of $S_{4}$ and $P$ a variable point of $\varsigma\left(P^{\prime}, \delta\right)$, then $F_{0}(\Pi, P)$ is constant. Hence, if a variable point $P$ is restricted to $\subseteq\left(P^{\prime}, \delta\right)$ then $F_{0}(\Pi, P)$ is a function of $\Pi$ alone throughout $S_{4}$. If $\Pi_{1}$ is a variable point of the sphere $S_{3}$ of radius $3 \delta$ with center at $P^{\prime}$, then the cube $\Re_{1}^{\prime}$ lies in the interior of $S_{4}$. Hence if $P$ is restricted to $\subseteq\left(P^{\prime}, \delta\right)$ then $F_{1}\left(\Pi_{1}, P\right)$ is a function of $\Pi_{1}$ alone throughout $S_{3}$.

We now define the function $\Phi_{1}\left(\Pi_{1}, P^{\prime}\right)$ for any point $P^{\prime}$ of $T$ and any point $\Pi_{1}$ in the interior of the corresponding sphere $S_{3}$ by requiring that, for any admissible $P^{\prime}, \Phi_{1}\left(\Pi_{1}, P^{\prime}\right)$ coincide with the function of $\Pi_{1}$ to which $F_{1}\left(\Pi_{1}, P\right)$ reduces when $P$ is restricted to $\varsigma\left(P^{\prime}, \delta\right)$. For fixed $P^{\prime}$ in $T, \Phi_{1}\left(\Pi_{1}, P^{\prime}\right)$ is a continuous function of $\Pi_{1}$ in $S_{3}$, and $\left|\Phi_{1}\left(\Pi_{1}, P^{\prime}\right)\right| \leqq M$. We also define a function $\Phi\left(P, P^{\prime}\right)$ for any point $P^{\prime}$ of $T$ and any point $P$ (not necessarily in $T$ ) in the interior of the sphere $S_{1}$ of radius $\delta$ with center at $P^{\prime}$, by setting $\Phi\left(P, P^{\prime}\right)=$

$\frac{1}{\delta^{6}} \int_{z-\delta / 2}^{z+\delta / 2} d \zeta_{2} \int_{y-\delta / 2}^{y+\delta / 2} d \eta_{2} \int_{x-\delta / 2}^{x+\delta / 2} d \xi_{2} \int_{\zeta_{2}-\delta / 2}^{\zeta_{2}+\delta / 2} d \zeta_{1} \int_{\eta_{2}-\delta / 2}^{\eta_{2}+\delta / 2} d \eta_{1} \int_{\xi_{2}-\delta / 2}^{\xi_{2}+\delta / 2} \Phi_{1}\left(\Pi_{1}, P^{\prime}\right) d \xi_{1}$.

Let $P^{\prime}$ be any fixed point of $T$. Then $\Phi\left(P, P^{\prime}\right)$ is a continuous function of $P$ throughout the interior of the sphere $S_{1}$, and $\left|\Phi\left(P, P^{\prime}\right)\right| \leqq M$; moreover $\Phi\left(P, P^{\prime}\right)$ coincides with $\mathscr{F}(P)$ throughout $\subseteq\left(P^{\prime}, \delta\right)$. 
We shall now prove that the function $\mathscr{F}(P)$ is pseudo-uniformly continuous in $T$. In order to do this, we shall show that, given any positive $\epsilon^{\prime}$, there exists a positive $\delta^{\prime}$, independent of the arbitrarily chosen point $P^{\prime}:\left(x^{\prime}, y^{\prime}, z^{\prime}\right)$ of $T$, such that

$$
\left|\mathfrak{F}(P)-\mathscr{F}\left(P^{\prime}\right)\right|<\epsilon^{\prime},
$$

for each point $P:(x, y, z)$ of $\subseteq\left(P^{\prime}, \delta^{\prime}\right)$. As a first restriction on $\delta^{\prime}$ we require that $\delta^{\prime}<3^{-1 / 2} \delta$. Then every point of $\subseteq\left(P^{\prime}, \delta^{\prime}\right)$ is a point of the cube

$\Re_{3}^{\prime}: x^{\prime}-\delta^{\prime} \leqq x \leqq x^{\prime}+\delta^{\prime}, \quad y^{\prime}-\delta^{\prime} \leqq y \leqq y^{\prime}+\delta^{\prime}, \quad z^{\prime}-\delta^{\prime} \leqq z \leqq z^{\prime}+\delta^{\prime}$, and every point of this cube is a point of the sphere $S_{1}$ with center at $P^{\prime}$ and radius $\delta$. Now if $P(x, y, z)$ is a point of $\Re_{3}^{\prime}$ then the points $\left(x^{\prime}, y, z\right)$, $\left(x^{\prime}, y^{\prime}, z\right)$ and $\left(x^{\prime}, y^{\prime}, z^{\prime}\right)$ are also points of this cube, and so we have*

Similarly,

$$
\left|\Phi\left(x, y, z ; x^{\prime}, y^{\prime}, z^{\prime}\right)-\Phi\left(x^{\prime}, y, z ; x^{\prime}, y^{\prime}, z^{\prime}\right)\right| \leqq \frac{2 M\left|x^{\prime}-x\right|}{\delta} \leqq \frac{2 M \delta^{\prime}}{\delta} .
$$

$$
\begin{gathered}
\left|\Phi\left(x^{\prime}, y, z ; x^{\prime}, y^{\prime}, z^{\prime}\right)-\Phi\left(x^{\prime}, y^{\prime}, z ; x^{\prime}, y^{\prime}, z\right)\right| \leqq \frac{2 M \delta^{\prime}}{\delta}, \\
\left|\Phi\left(x^{\prime}, y^{\prime}, z ; x^{\prime}, y^{\prime}, z^{\prime}\right)-\Phi\left(x^{\prime}, y^{\prime}, z^{\prime} ; x^{\prime}, y^{\prime}, z^{\prime}\right)\right| \leqq \frac{2 M \delta^{\prime}}{\delta} .
\end{gathered}
$$

Adding these inequalities we obtain

$$
\left|\Phi\left(P, P^{\prime}\right)-\Phi\left(P^{\prime}, P^{\prime}\right)\right| \leqq \frac{6 M \dot{\delta}^{\prime}}{\delta},
$$

for an arbitrary point $P$ of $\Re_{3}^{\prime}$. We infer that $\left|\mathcal{F}(P)-\mathscr{F}\left(P^{\prime}\right)\right| \leqq 6 M \delta^{\prime} / \delta$, throughout $\subseteq\left(P^{\prime}, \delta^{\prime}\right)$. If we choose a positive $\delta^{\prime}$ less than each of the quantities $3^{-1 / 2} \delta$ and $M^{-1} \delta \epsilon^{\prime} / 6$, then $\left|\mathcal{F}(P)-\mathcal{F}\left(P^{\prime}\right)\right|<\epsilon^{\prime}$ for every point $P$ of $\subseteq\left(P^{\prime}, \delta^{\prime}\right)$. Since the restrictions imposed on $\delta^{\prime}$ are independent of the position of $P^{\prime}$ in $T$ this means that $\mathcal{F}(P)$ is pseudo-uniformly continuous in $T$.

It remains to establish item (2) of the conclusion of the theorem. That $\mathscr{F}(P)$ has continuous partial derivatives of the first and second order in the neighborhood of an arbitrarily chosen point $P^{\prime}$ of $T$ becomes obvious when we replace $\mathcal{F}(P)$ by $\Phi\left(P, P^{\prime}\right)$ in $\subseteq\left(P^{\prime}, \delta\right)$. We have, in particular, the following formula, valid in $\subseteq\left(P^{\prime}, \delta\right)$ :

$$
\begin{aligned}
\frac{\partial^{2}}{\partial x^{2}} \mathscr{F}(P)= & \frac{1}{\delta^{6}} \int_{z-\delta / 2}^{z+\delta / 2} d \zeta_{2} \int_{y-\delta / 2}^{y+\delta / 2} d \eta_{2} \int_{\zeta_{2}-\delta / 2}^{\zeta_{2}+\delta / 2} d \zeta_{1} \int_{\eta_{2}-\delta / 2}^{\eta_{2}+\delta / 2}\left\{\Phi_{1}\left(x+\delta, \eta_{1}, \zeta_{1} ; x^{\prime}, y^{\prime}, z^{\prime}\right)\right. \\
& \left.-2 \Phi_{1}\left(x, \eta_{1}, \zeta_{1} ; x^{\prime}, y^{\prime}, z^{\prime}\right)+\Phi_{1}\left(x-\delta, \eta_{1}, \zeta_{1} ; x^{\prime}, y^{\prime}, z^{\prime}\right)\right\} d \eta_{1} .
\end{aligned}
$$

* It will be convenient in some cases to replace the symbol $\Phi\left(P, P^{\prime}\right)$ by $\Phi\left(x, y, z ; x^{\prime}, y^{\prime}, z^{\prime}\right)$. Similarly, $\Phi_{1}\left(P, P^{\prime}\right)$ may be replaced by $\Phi_{1}\left(x, y, z ; x^{\prime}, y^{\prime}, z^{\prime}\right)$. 
From this we have

$$
\left|\frac{\partial^{2}}{\partial x^{2}} \mathcal{F}(P)\right|_{P=P^{\prime}} \leqq \frac{4 M}{\delta^{2}}
$$

Similarly,

$$
\left|\frac{\partial^{2}}{\partial y^{2}} \mathcal{F}(P)\right|_{P=P^{\prime}} \leqq \frac{4 M}{\delta^{2}} \text {, and }\left|\frac{\partial^{2}}{\partial z^{2}} \mathscr{F}(P)\right|_{P=P^{\prime}} \leqq \frac{4 M}{\delta^{2}}
$$

Since $P^{\prime}$ is an arbitrarily chosen point of $T$, we infer that

$$
\left|\nabla^{2} \mathfrak{F}(P)\right| \leqq \frac{12 M}{\delta^{2}}
$$

throughout $T$. This completes the proof of the theorem.

\section{ORDINARY DOMAINS}

We now consider briefly a special type of finite domain.

Definition 23. A finite domain $T$ is said to be ordinary if every function of the variable point $P$ of $T$ which approaches the value zero pseudo-continuously at each element $\gamma$ of $t$ approaches the boundary values $f(\gamma)=0$ on $t$ with uniform pseudo-continuity.

THEOREM 12. Let $f(\gamma)$ be any bounded and uniformly pseudo-continuous function of the variable element $\gamma$ of the boundary of an ordinary domain $T$. Then any function $F(P)$ which approaches the boundary values $f(\gamma)$ with pseudo-continuity approaches these boundary values with uniform pseudo-continuity.

From Theorem 9 we know that there exists in $T$ a function $F_{1}(P)$ which approaches the boundary values $f(\gamma)$ with uniform pseudo-continuity. Now the difference between the functions $F(P)$ and $F_{1}(P)$ approaches the value zero pseudo-continuously at each element of $t$; therefore (since $T$ is ordinary) this difference vanishes on $t$ with uniform pseudo-continuity. It follows that $F(P)$ approaches the boundary values $f(\gamma)$ with uniform pseudo-continuity.

THEOREM 13. There exist finite domains $T$, having only accessible boundary points, which are not ordinary.

We shall prove this theorem by exhibiting an example. Consider the domain $T$ which is constructed by deleting from the open sphere

$$
x^{2}+y^{2}+z^{2}<1
$$

all points which lie on the following surfaces: 
(1) $x^{2}+y^{2}=\frac{1}{4}$,

$$
z=0 \text {, }
$$$$
z=\frac{1}{i+2}, \quad \frac{1}{(i+2)^{2}} \leqq x^{2}+y^{2} \leqq \frac{1}{4}, \quad \text { where } i=1,2,3, \cdots \text {. }
$$

Consider now the function $F(P)$ defined at each point $P:(x, y, z)$ of $T$ requiring that when

$$
x^{2}+y^{2}<\frac{1}{4}, \text { and } 0<z \leqq \frac{1}{2}
$$

we have

$$
F(P)=\left(x^{2}+y^{2}\right)\left(\frac{1}{4}-x^{2}-y^{2}\right) \sin \frac{\pi}{z},
$$

while at all other points of $T$ we have $F(P)=0$. The function $F(P)$ approaches the value zero at each boundary element. But $F(P)$ does not approach the boundary value zero with uniform pseudo-continuity, for if it did we could infer from Theorem 10 that $F(P)$ approached zero continuously on the boundary, which would be inconsistent with the behavior of $F(P)$ at the points

$$
P_{j}: \quad\left(\frac{1}{3}, \frac{1}{3}, \frac{2}{2 j+5}\right) \quad(j=1,2,3, \cdots) \text {. }
$$

THEOREM 14. If there exists a function $\alpha(p)$ having a positive value at each point $p$ of $t$ and such that every sphere with center at any point $p$ of $t$ and radius less than $\alpha(p)$ yields, as the subset of its interior points which belong to $T$, a finite sum of finite domains, then $T$ is an ordinary domain.

Suppose that there exists a finite domain, $T$, which satisfies the hypothesis of the theorem but is not ordinary. Then there exists in $T$ a function $F(P)$ which approaches the boundary values $f(\gamma)=0$ on $t$ with non-uniform pseudo-continuity. There exists a positive constant $\epsilon$ such that the set $E$ of all points of $T$ at which $|F(P)| \geqq \epsilon$ has at least one limit point on $t$. Let $\bar{p}$ be any such point of $t$. Let $S_{1}$ be a sphere with center at $\bar{p}$ and radius $\alpha(\bar{p}) / 2$. The set of points common to $T$ and the interior of $S_{1}$ consists of a finite sum of finite domains, of which at least one has $\bar{p}$ as a limit point. Among those which have $\bar{p}$ as a limit point, at least one contains infinitely many points of $E$. We choose arbitrarily a Cartesian coordinate system; it is then possible to specify uniquely* a particular domain, $G_{1}$, having these properties.

* Consider the point set comprising all points belonging to domains eligible for choice as $G_{1}$. A variable point of this set attains its maximum distance from the boundary of this set at one or more fixed points of which a particular one, $Q$, may be specified by the method explained in connection with the proof of Theorem 6. We select as $G_{1}$ that eligible domain which contains $Q$. 
We now replace $S_{1}$ by a concentric sphere $S_{2}$ of radius $\alpha(\bar{p}) / 2^{2}$. The set of points common to $G_{1}$ and the interior of $S_{2}$ consists of a finite sum of finite domains of which at least one has $\bar{p}$ as a limit point, and contains infinitely many points of $E$. As before we may select uniquely (relative to the previously chosen coordinate system) a particular domain $G_{2}$ having these properties.

Proceeding in this way we obtain an infinite sequence of finite domains: $G_{1}, G_{2}, G_{3}, \cdots$. There exists a positive integer $k$ such that for all integers $i$ greater than $k, G_{i}$ is a partial domain of $T$. Denoting by $G_{i}^{\prime}$ the closed partial region of $T^{\prime}$ corresponding to the partial domain $G_{i}$ we readily verify that the sequence of closed partial regions of $T^{\prime}: G_{k+1}{ }^{\prime}, G_{k+2}{ }^{\prime}, G_{k+3}{ }^{\prime}, \cdots$ is monotone, and so determines a component of $T^{\prime}$. Since the diameter of $G_{i}^{\prime}$ is at most $\alpha(\bar{p}) / 2^{i-1}$ this component is an element $\bar{\gamma}$ of $t$.

Every pseudo-spherical domain corresponding to $\bar{\gamma}$ contains points of $E$, at which $|F(P)| \geqq \epsilon$. Hence $F(P)$ does not approach the value zero at $\bar{\gamma}$. This is inconsistent with the hypothesis of the theorem, and so establishes the falsity of the assumption that there exists a finite domain $T$, satisfying the condition given in the theorem, which is not ordinary.

\section{An extension of the Dirichlet problem}

\section{The pseudo-classical Dirichlet problem}

We first consider the following proposition:

THEOREM 15. Given a bounded and uniformly pseudo-continuous function $f(\gamma)$ of a variable element $\gamma$ of the boundary of a finite domain $T$, there exists at most one function $U(P)$ of the variable point $P$ of $T$ which is single-valued and harmonic throughout $T$ and approaches the boundary values $f(\gamma)$ with uniform pseudo-continuity.

The difference between any two functions which satisfy the conditions imposed on $U(P)$ is a harmonic function in $T$, and approaches zero continuously on $t$. Such a function is identically zero in $T$, and so the two given functions are identical.

DeFINITION 24. Let $f(\gamma)$ be a bounded and uniformly pseudo-continuous function of the variable element $\gamma$ of the boundary of a finite domain $T$. If there exists a function $U(P)$ of the variable point $P$ of $T$ which is harmonic in $T$ and approaches the boundary values $f(\gamma)$ with uniform pseudo-continuity, then this function $U(P)$ is called the solution of the Dirichlet problem, in the pseudoclassical sense, for the domain $T$ and the boundary values $f(\gamma)$.

We note that (by virtue of Theorem 10) a solution of the Dirichlet prob- 
lem in the classical sense for continuous boundary values $\phi(p)$ on $t$ may be regarded as a solution in the pseudo-classical sense for boundary values defined by the corresponding function $f(\gamma)$ of the variable element $\gamma$ of $t$.

DEFINITION 25. If a finite domain $T$ is such that for every bounded and uniformly pseudo-continuous function $f(\gamma)$ of a variable element $\gamma$ of $t$ there exists a solution of the Dirichlet problem in the pseudo-classical sense for $T$ and $f(\gamma)$, then $T$ is said to be pseudo-normal.

A pseudo-normal finite domain is necessarily normal, i.e., one for which the Dirichlet problem is possible in the classical sense.

THEOREM 16. Let $U(P)$ be a function which is harmonic in $T$ and approaches, with uniform pseudo-continuity, boundary values $f(\gamma)$ such that

$$
c_{1} \leqq f(\gamma) \leqq c_{2},
$$

on $t$, where $c_{1}$ and $c_{2}$ are constants. Then

$$
c_{1} \leqq U(P) \leqq c_{2},
$$

throughout $T$; moreover if $U(P)$ is not a constant we have

throughout $T$.

$$
c_{1}<U(P)<c_{2},
$$

Suppose that there exists a point $Q$ of $T$ such that $U(Q)>c_{2}$. Then there exists a quantity $\delta$, independent of the arbitrarily chosen element $\gamma_{1}$ of $t$, such that

$$
\left|U(P)-f\left(\gamma_{1}\right)\right|<\frac{U(Q)-c_{2}}{2},
$$

at each point $P$ of $\subseteq\left(\gamma_{1}, \delta\right)$. Therefore at each point $P^{\prime}$ of $T$ whose distance from $t$ is less than $\delta$,

$$
U\left(P^{\prime}\right)<\frac{U(Q)+c_{2}}{2}<U(Q) .
$$

Consider the set $E$ of points of $T$ at which

$$
U(P)>\frac{U(Q)+c_{2}}{2} .
$$

Now $Q$ is an interior point of $E$, and the set $\mathcal{T}$ containing each point of $E$ which can be joined to $Q$ by a continuous curve lying in $E$ is a finite domain. Each boundary point of $\tau$ is at a distance at least $\delta$ from $t$. Hence $U(P)$ is continuous on the boundary of $\mathcal{T}$; moreover it has there the constant value $\left[U(Q)+c_{2}\right] / 2$. But in the interior of $\mathcal{T}$ the function $U(P)$ is harmonic and 


$$
U(P)>\frac{U(Q)+c_{2}}{2}
$$

This inconsistency establishes the falsity of the assumption that there exists a point $Q$ of $T$ such that $U(Q)>c_{2}$. Similarly it may be shown that there exists no point $Q^{\prime}$ of $T$ such that $U\left(Q^{\prime}\right)<c_{1}$.

Suppose that $T$ contains a point $Q_{1}$ such that $U\left(Q_{1}\right)=c_{1}$. Let $P_{1}$ be any other point of $T$, and let $\tau_{1}$ be a domain which contains $P_{1}$ and $Q_{1}$ and lies (together with its boundary) in the interior of $T$. From a well known property of harmonic functions we see that $U(P)=c_{1}$ throughout $\tau_{1}$. Hence $U\left(P_{1}\right)=c_{1}$, and since $P_{1}$ is an arbitrary point of $T$, we infer that $U(P)$ is constant in $T$. Similarly if $T$ contains a point $Q_{2}$ such that $U\left(Q_{2}\right)=c_{2}$, then $U(P)$ is constant in $T$.

\section{SUBHARMONIC AND SUPERHARMONIC FUNCTIONS}

In the discussions of some of the theorems given below, we shall have frequent occasion to use properties of functions which are continuous and subharmonic or superharmonic in $T$. We use these terms in a sense similar to that adopted by Kellogg*:

Definition 26. Let $W(P)$ be a function which is continuous in T. Let $\tau$ be a finite domain contained (together with its boundary) in $T$, and let $u(P)$ be a function which is harmonic in $\mathcal{T}$ and continuous on the boundary of $\mathcal{T}$. If for every $\mathcal{T}$ and every $u(P)$ such that $W(P) \leqq u(P)$ on the boundary of $\mathcal{T}$ we have $W(P) \leqq u(P)$ in the interior of $\mathcal{T}$, then $W(P)$ is said to be subharmonic in $T$; if, on the other hand, for every $\mathcal{T}$ and every $u(P)$ such that $W(P) \geqq u(P)$ on the boundary of $\mathcal{T}$ we have $W(P) \geqq u(P)$ in the interior of $\mathcal{T}$, then $W(P)$ is said to be superharmonic in $T$.

THEOREM 17. Let $W(P)$ be a continuous superharmonic function of the variable point $P$ of $T$. If to every positive constant $\epsilon$ there corresponds a positive constant $\delta$, independent of $P$, such that $-\epsilon<W(P)$ for each point $P$ of $T$ which is at a distance less than $\delta$ from $t$, then $0 \leqq W(P)$ throughout $T$.

Let $\bar{P}$ be an arbitrarily chosen fixed point of $T$. There exists a domain $\tau$ containing $\bar{P}$, which lies in $T$ and has a boundary each point of which is at a distance from $t$ which is positive and less than the $\delta$ corresponding to a preassigned positive $\epsilon$. Now the function $W_{1}(P)=W(P)+\epsilon$ is superharmonic in $\mathcal{T}$ and has a positive minimum on the boundary of $\mathcal{T}$, and therefore a positive lower bound in the interior of $\mathcal{T}$. It follows that $-\epsilon<W(\bar{P})$. Since $\epsilon$ is

* O. D. Kellogg, loc. cit., p. 315 ff. Kellogg formulates the definition for a region $R$, which may be interpreted as a domain. We shall need some of the properties given in Kellogg's treatment of these functions. 
any positive number, and $\bar{P}$ any point of $T$, we infer that $0 \leqq W(P)$ throughout $T$.

COROLlaRy. Let $W(P)$ be a bounded, superharmonic and pseudo-uniformly continuous function in $T$, determining boundary values* $f(\gamma)$ on $t$. If $0 \leqq f(\gamma)$ on $t$, then $0 \leqq W(P)$ throughout $T$.

THEOREM 18. Let $W(P)$ be a function which is bounded and pseudo-uniformly continuous in $T$. Let $\mathcal{T}$ be a domain (containing $\dagger$ only points of $T$ ) such that there exists a function $u(P)$ which is harmonic in $\mathcal{T}$ and approaches with uniform pseudo-continuity the same boundary values on the boundary of $\mathcal{T}$ as does $W(P)$. Let $\mathfrak{W}(P)$ be the function which coincides with $u(P)$ in $\mathcal{T}$ and with $W(P)$ in $T-\mathcal{T}$. Then $\mathscr{W}(P)$ is bounded and pseudo-uniformly continuous in $T$ and approaches with pseudo-uniform continuity the same boundary values on $t$ as does $W(P)$. Moreover, if $W(P)$ is superharmonic in $T$, then $\mathscr{W}(P)$ is superharmonic in $T$, and $W(P) \leqq W(P)$ in $T$.

From Theorem 10 we see that the function $w(P)=W(P)-\mathscr{W}(P)$ approaches the boundary value zero continuously on the boundary of $\mathcal{T}$ and vanishes identically in $T-\tau$. Hence $w(P)$ is uniformly continuous on $T+t$ (when defined as zero on $t$ ) and therefore is bounded and pseudo-uniformly continuous in $T$. Hence $\mathscr{W}(P)=W(P)-w(P)$ is also bounded and pseudo-uniformly continuous in $T$, and approaches with pseudo-uniform continuity the same boundary values on $t$ as does $W(P)$.

If $W(P)$ is superharmonic in $T$, then it follows from the Corollary of Theorem 17 that $\mathscr{W}(P) \leqq W(P)$ in $\mathcal{T}$, and so in $T$. That $\mathscr{W}(P)$ is superharmonic in $T$ can be proved by the same reasoning as that given by Kellogg $\ddagger$ in the demonstration of an analogous theorem: the value of $\mathscr{W}(P)$ at any point $Q$ of $T$ is readily shown to be greater than or equal to the average value of $\mathscr{W}(P)$ on the surface of any sufficiently small sphere with center at $Q$.

THEOREM 19. Let $F(P)$ be a function which is bounded and pseudo-uniformly continuous in $T$, and let $\epsilon$ be any positive constant. Then there exists a function $\mathfrak{F}(P)$ which has the following properties:

(1) The function $\mathcal{F}(P)$ may be expressed as the difference of two functions, each of which is bounded, pseudo-uniformly continuous, and subharmonic in $T$.

(2) The inequality $|\mathcal{F}(P)-F(P)|<\epsilon$ holds throughout $T$.

\footnotetext{
* That $W(P)$ approaches (with uniform pseudo-continuity) bounded and uniformly pseudocontinuous boundary values on $t$, is evident from Theorem 8 .

$\dagger$ It should be noted that in this theorem there is no restriction that the boundary of $\mathcal{T}$ should be contained in $T$.

$\ddagger$ O. D. Kellogg, loc. cit., p. 317. (Proof of property 4 of superharmonic functions.)
} 
We introduce a Cartesian coordinate system; then the function $\mathscr{F}(P)$, the existence of which has been established in Theorem 11, has the required properties. This function satisfies the relation

$$
|\mathfrak{F}(P)-F(P)|<\epsilon
$$

throughout $T$. Furthermore there exists a positive constant $K$ such that $\left|\nabla^{2} \mathcal{F}(P)\right|<K$, throughout $T$.

Let $Q$ be an arbitrarily chosen fixed point exterior to $T$, and denote by $r_{0}$ the maximum distance from $Q$ to a point of $t$, and by $r$ the distance from $Q$ to the variable point $P$ of $T$. We set* $\mathcal{F}(P)=\mathcal{F}^{\prime}(P)-\mathcal{F}^{\prime \prime}(P)$ where

$$
F^{\prime}(P)=\mathscr{F}(P)+\frac{K r_{0}}{2} r, \text { and } \mathcal{F}^{\prime \prime}(P)=\frac{K r_{0}}{2} r .
$$

The functions $\mathcal{F}^{\prime}(P)$ and $\mathcal{F}^{\prime \prime}(P)$ are pseudo-uniformly continuous in $T$, and have continuous partial derivatives of the first and second order, throughout $T$. Moreover at any point $P$ of $T$

$$
\nabla^{2} \mathcal{F}^{\prime}(P)=\nabla^{2} \mathfrak{F}(P)+\frac{K r_{0}}{r}>0, \text { and } \nabla^{2} \mathcal{F}^{\prime \prime}(P)=\frac{K r_{0}}{r}>0 .
$$

This shows that $\mathcal{F}^{\prime}(P)$ and $\mathcal{F}^{\prime \prime}(P)$ are subharmonic in $T$.

Definition 27. By $\Psi(\gamma, P)^{\circ}$, the pseudo-distance from an element $\gamma$ of $t$ to a point $P$ of $T$, we mean the least upper bound of the radii of all pseudo-spherical domains corresponding to $\gamma$ which do not contain $P$.

THEOREM 20. If $\gamma_{1}$ is a fixed element of $t$ and $P$ a variable point of $T$, then $\Psi\left(\gamma_{1}, P\right)$ has the following properties:

(1) $\Psi\left(\gamma_{1}, P\right)$ is a bounded and pseudo-uniformly continuous function of $P$ throughout $T$.

(2) In the part of $T$ outside any given pseudo-spherical domain (not identical with $T)$ corresponding to $\gamma_{1}, \Psi\left(\gamma_{1}, P\right)$ has a positive lower bound.

(3) $\Psi\left(\gamma_{1}, P\right)$ approaches (with uniform pseudo-continuity) bounded and uniformly pseudo-continuous boundary values $\psi(\gamma)$ such that $\psi(\gamma)>0$ on $t$, except at $\gamma=\gamma_{1}$, and $\psi\left(\gamma_{1}\right)=0$.

(4) $\Psi\left(\gamma_{1}, P\right)$ is subharmonic in $T$.

We denote by $r$ the distance from the point $p_{1}$ contained in $\gamma_{1}$ to the variable point $P$ of $T$. Let $\rho$ be any constant such that $\subseteq\left(\gamma_{1}, \rho\right)$ is not identical with $T$. Then $\rho<\bar{r}$, where $\bar{r}$ is the least upper bound of $r$ in $T$. If $P$ is a point of $\mathfrak{S}\left(\gamma_{1}, \rho\right)$, and therefore a point of $\mathfrak{S}\left(\gamma_{1}, \rho^{\prime}\right)$ for some $\rho^{\prime}$ less than $\rho$, then

\footnotetext{
* In the corresponding problem in two dimensions we set $\mathcal{F}^{\prime}(P)=\mathscr{F}(P)+K r_{0} r$ and $\mathcal{F}^{\prime \prime}(P)=K r_{0}$.
} 


$$
\Psi\left(\gamma_{1}, P\right)<\rho .
$$

If $P$ is a point of $T-\Im\left(\gamma_{1}, \rho\right)$, then

$$
\rho \leqq \Psi\left(\gamma_{1}, P\right)<\bar{r} .
$$

These relations show that $\Psi\left(\gamma_{1}, P\right)$ has property (2), and also that $\Psi\left(\gamma_{1}, P\right)$ is bounded, and approaches pseudo-continuously the value zero at the boundary element $\boldsymbol{\gamma}_{1}$.

The function $\Psi\left(\gamma_{1}, P\right)$ is pseudo-uniformly continuous in $T$. To prove this we shall show that, given any positive $\epsilon$, the relation

$$
\left|\Psi\left(\gamma_{1}, P^{\prime}\right)-\Psi\left(\gamma_{1}, P^{\prime \prime}\right)\right|<\epsilon
$$

holds for every pair of points $P^{\prime}$ and $P^{\prime \prime}$ that can be joined by a continuous curve in $T$, the maximum distance between two points of which is less than $\epsilon / 3$. Given such a pair of points, $P^{\prime}$ belongs to

$$
\Im\left(\gamma_{1}, \Psi\left(\gamma_{1}, P^{\prime}\right)+\frac{\epsilon}{3}\right),
$$

and consequently $P^{\prime \prime}$ belongs to

$$
\Im\left(\gamma_{1}, \Psi\left(\gamma_{1}, P^{\prime}\right)+\frac{2 \epsilon}{3}\right) \text {. }
$$

We infer that

$$
\Psi\left(\gamma_{1}, P^{\prime \prime}\right)<\Psi\left(\gamma_{1}, P^{\prime}\right)+\epsilon
$$

Similarly,

$$
\Psi\left(\gamma_{1}, P^{\prime}\right)<\Psi\left(\gamma_{1}, P^{\prime \prime}\right)+\epsilon
$$

whence,

$$
\left|\Psi\left(\gamma_{1}, P^{\prime}\right)-\Psi\left(\gamma_{1}, P^{\prime \prime}\right)\right|<\epsilon .
$$

Since $\Psi\left(\gamma_{1}, P\right)$ is bounded and pseudo-uniformly continuous in $T$, it approaches, with uniform pseudo-continuity, bounded and pseudo-uniformly continuous boundary values $\psi(\gamma)$ on $t$. That $\psi(\gamma)>0$ on $t$, except at $\gamma=\gamma_{1}$ where $\psi(\gamma)=0$, is immediately obvious from properties of $\Psi\left(\gamma_{1}, P\right)$ already established.

In order to show that $\Psi\left(\gamma_{1}, P\right)$ is subharmonic in $T$, we now define a function $\Omega\left(\gamma_{1}, \rho, P\right)$ for every positive value of $\rho$ and every point $P$ of $T$. If $P$ is a point of $T$ for which $r<\rho$, but which does not belong to $\subseteq\left(\gamma_{1}, \rho\right)$, then $\Omega\left(\gamma_{1}, \rho, P\right)=\rho$; for every other point $P$ of $T$ we set $\Omega\left(\gamma_{1}, \rho, P\right)=r$. If $\rho$ is held fast then $\Omega\left(\gamma_{1}, \rho, P\right)$ is a continuous function of $P$ in $T$, subharmonic in a 
sufficiently small neighborhood of each point of $T$, and therefore subharmonic* in $T$. Moreover at each point $P$ of $T$ the least upper bound of $\Omega\left(\gamma_{1}\right.$, $\rho, P)$ for all positive $\rho$ is $\Psi\left(\gamma_{1}, P\right)$.

Let $\tau$ be any finite domain, contained (together with its boundary) in $T$. Let $u(P)$ be any function harmonic in $\mathcal{T}$ such that

$$
u(P) \geqq \Psi\left(\gamma_{1}, P\right)
$$

on the boundary of $\tau$. Then

$$
u(P) \geqq \Omega\left(\gamma_{1}, \rho, P\right),
$$

for any $\rho>0$. Since $\Omega\left(\gamma_{1}, \rho, P\right)$ is subharmonic in $T$ the second relation holds in the interior as well as on the boundary of $\mathcal{T}$. Inasmuch as $\Psi\left(\gamma_{1}, P\right)$ is the least upper bound of $\Omega\left(\gamma_{1}, \rho, P\right)$ for all $\rho>0$, we conclude that

$$
u(P) \geqq \Psi\left(\gamma_{1}, P\right),
$$

throughout the interior of $\mathcal{T}$. Hence $\Psi\left(\gamma_{1}, P\right)$ is a subharmonic function of $P$ throughout $T$.

\section{The sequence solution of the extended Dirichlet problem}

Definition 28. An infinite sequence of finite domains, $\left\{T_{i}\right\}$ :

$$
T_{1}, T_{2}, T_{3}, \cdots,
$$

is said to be a pseudo-normal sequence $\dagger$ of domains in $T$ if the following conditions are satisfied:

(1) Each $T_{i}$ is contained in $T$.

(2) Each point of $T$ is the center of a sphere which is contained in infinitely many of the domains $T_{i}$.

(3) Given any domain $T_{i}$ and any function $F(P)$, bounded and pseudo-uniformly continuous in $T$, there exists a function which is harmonic in $T_{i}$ and approaches (with uniform pseudo-continuity) the same boundary values on the boundary of $T_{i}$ as does $F(P)$.

Condition (3) is satisfied (for a $T_{i}<T$ ) if $T_{i}$ is pseudo-normal, or if $T_{i}$ is normal and has a boundary contained in $T$. Given any finite domain $T$ there exist pseudo-normal sequences of domains in $T$, for any nested sequence of normal domains approximating to $T$ satisfies all the conditions given above.

\footnotetext{
* We use here theorems relating to subharmonic functions analogous to those given with respect to superharmonic functions by Kellogg (loc. cit.) in the exercise on p. 317 , and in property 2 on p. 316 .

$\dagger$ Such a sequence corresponds to the sequence of closed regions $R_{1}, R_{2}, R_{3}, \cdots$, used by Kellogg (loc. cit., p. $322 \mathrm{ff}$.) in the study of the sequence solution of the generalized Dirichlet problem.
} 
Definition 29. By the sequence of functions $\left\{U_{i}(P)\right\}: U_{0}(P), U_{1}(P)$, $U_{2}(P), \cdots$, associated with $T,\left\{T_{i}\right\}$ (a given pseudo-normal sequence of domains in $T$ ), and $F(P)$ (a given function, bounded and pseudo-uniformly continuous in $T$ ), we mean the infinite sequence of functions uniquely determined* by the following conditions:

(1) $U_{0}(P)$ is identical in $T$ with $F(P)$.

(2) For every positive integral $i, U_{i}(P)$ is identical in $T-T_{i}$ with $U_{i-1}(P)$, and in $T_{i}$ with that function which is harmonic in $T_{i}$ and approaches (with uniform pseudo-continuity) the same values on the boundary of $T_{i}$ as does $U_{i-1}(P)$.

THEOREM 21. Given a bounded and uniformly pseudo-continuous function $f(\gamma)$, defined on the boundary of a finite domain $T$, let $\left\{T_{i}\right\}$ be an arbitrarily chosen pseudo-normal sequence of domains in $T$, and let $F(P)$ be any function which is bounded and continuous in $T$ and approaches the boundary values $f(\gamma)$ with uniform pseudo-continuity. Then the sequence of functions $\left\{U_{i}(P)\right\}$ associated with $T,\left\{T_{i}\right\}$ and $F(P)$ converges in $T$ (uniformly in any closed region contained in $T)$ to a function $U(P)$ which is harmonic in $T$ and depends only on $T$ and $f(\gamma)$.

Let $\epsilon$ be an arbitrarily chosen positive constant. By Theorem 19, there exist functions $\mathscr{F}(P), \mathcal{F}^{\prime}(P)$ and $\mathscr{F}^{\prime \prime}(P)$ such that

$$
\mathscr{F}(P)=\mathscr{F}^{\prime}(P)-\mathscr{F}^{\prime \prime}(P),
$$

where $\mathcal{F}^{\prime}(P)$ and $\mathcal{F}^{\prime \prime}(P)$ are each bounded, pseudo-uniformly continuous and subharmonic in $T$, and

$$
|\mathscr{F}(P)-F(P)|<\frac{\epsilon}{3} .
$$

Let $\left\{U_{i}^{\prime}(P)\right\}$ be the sequence of functions associated with $T,\left\{T_{i}\right\}$, and $\mathcal{F}^{\prime}(P)$. Now Theorem 18 implies that throughout $T$ each $U_{i}^{\prime}(P)$ is subharmonic, and $\left\{U_{i}^{\prime}(P)\right\}$ is a monotone increasing sequence of functions. Using Theorem 16 we infer that for each non-negative integral $i$, the least upper bound of $U_{i+1}^{\prime}(P)$ is no greater than that of $U_{i}^{\prime}(P)$, and so we have $U_{i}^{\prime}(P) \leqq \mathcal{H}^{\prime}$ where $\mathcal{H}^{\prime}$ is the least upper bound of $U_{0}^{\prime}(P)=\mathcal{F}^{\prime}(P)$. Hence the sequence $\left\{U_{i}^{\prime}(P)\right\}$ converges, throughout $T$.

Any given point $P$ of $T$ is the center of a sphere $S$ which lies in infinitely many of the domains $T_{i}$. Hence the sequence $\left\{U_{i}^{\prime}(P)\right\}$ contains a bounded and monotone increasing subsequence of functions which are harmonic within $S$. The convergence of this subsequence, and so also the convergence of the

* Using the first part of Theorem 18 , it is readily seen by mathematical induction that each $U_{i-1}(P)$ determines uniquely a bounded and pseudo-uniformly continuous $U_{\boldsymbol{i}}(P)$. 
monotone sequence $\left\{U_{i}^{\prime}(P)\right\}$, is uniform* within $S$. By the Heine-Borel theorem we infer that $\left\{U_{i}{ }^{\prime}(P)\right\}$ converges uniformly in any closed region contained in $T$. Moreover the limiting function, $U^{\prime}(P)$, is harmonic in $T$.

Let $\left\{\mathfrak{I}_{i}\right\}$ be another pseudo-normal sequence of domains in $T$, and let $\left\{\mathfrak{u}_{i}^{\prime}(P)\right\}$ be the sequence of functions associated with $T,\left\{\mathfrak{I}_{i}\right\}$, and $\mathcal{F}^{\prime}(P)$. For any non-negative integral $i$ we may write

$$
U^{\prime}(P)-\mathfrak{u}_{i+1}^{\prime}(P)=\left[U^{\prime}(P)-\mathfrak{u}_{i}^{\prime}(P)\right]+\left[\mathfrak{u}_{i}^{\prime}(P)-\mathfrak{u}_{i+1}^{\prime}(P)\right] .
$$

Now $\left[\mathfrak{u}_{i}^{\prime}(P)-\mathfrak{U}_{i+1}^{\prime}(P)\right]$ approaches the value zero continuously on $t$. Hence if

$$
0 \leqq U^{\prime}(P)-\mathfrak{u}_{i}^{\prime}(P)
$$

throughout $T$, then to any preassigned positive $\epsilon$ there corresponds a positive $\delta$ independent of $P$ such that

$$
-\epsilon<U^{\prime}(P)-\mathfrak{u}_{i+1}^{\prime}(P),
$$

at each point $P$ of $T$ which lies at a distance less than $\delta$ from $t$. Using Theorem 17 , we infer that if

$$
0 \leqq U^{\prime}(P)-\mathfrak{H}_{i}^{\prime}(P)
$$

in $T$, then

$$
0 \leqq U^{\prime}(P)-\mathfrak{u}_{i+1}^{\prime}(P),
$$

in $T$. Since

$$
0 \leqq U^{\prime}(P)-\mathfrak{H}_{0}^{\prime}(P),
$$

we see by mathematical induction that throughout $T$,

$$
0 \leqq U^{\prime}(P)-\mathfrak{U}_{i}^{\prime}(P) \quad(i=0,1,2, \cdots) .
$$

Denoting by $\mathfrak{u}^{\prime}(P)$ the limit of thesequence $\left\{\mathfrak{u}_{i}^{\prime}(P)\right\}$, we have $\mathfrak{u}^{\prime}(P) \leqq U^{\prime}(P)$. Similarly, $U^{\prime}(P) \leqq \mathfrak{u}^{\prime}(P)$. Hence $\mathfrak{u}^{\prime}(P)=U^{\prime}(P)$, in $T$, and we conclude that the limit of the sequence $\left\{U_{i}^{\prime}(P)\right\}$ is independent of $\left\{T_{i}\right\}$.

The sequence $\left\{U_{i}^{\prime \prime}(P)\right\}$ associated with $T,\left\{T_{i}\right\}$, and $\mathcal{F}^{\prime \prime}(P)$ has similar properties. Furthermore, if we represent by $\left\{U_{i}(P)\right\}$ the sequence of functions associated with $T,\left\{T_{i}\right\}$, and $\mathcal{F}(P)$, then

$$
U_{i}(P)=U_{i}^{\prime}(P)-U_{i}^{\prime \prime}(P) \quad(i=0,1,2, \cdots) .
$$

Hence the sequence $\left\{U_{i}(P)\right\}$ also converges (uniformly in any closed region contained in $T$ ) to a function $U(P)$ which is harmonic in $T$, and independent of $\left\{T_{i}\right\}$.

* This follows from Harnack's second convergence theorem: A. Harnack, Grundlagen der Theorie des Logarithmischen Potentials, Leipzig, 1887, p. 67. 
Let $\left\{U_{i}(P)\right\}$ be the sequence associated with $T,\left\{T_{i}\right\}$, and $F(P)$. Then the sequence associated with $T,\left\{T_{i}\right\}$, and $\mathcal{F}(P)-F(P)$ is

$$
\left\{U_{i}(P)-U_{i}(P)\right\}: U_{0}(P)-U_{0}(P), U_{1}(P)-U_{1}(P), U_{2}(P)-U_{2}(P), \cdots .
$$

Hence,

$$
\left|U_{i}(P)-U_{i}(P)\right|<\epsilon / 3 \quad(i=0,1,2, \cdots) .
$$

For a bound for the first term of a pseudo-normal sequence is a bound for every term.

Now given any finite closed region $\mathcal{T}^{\prime}$ contained in $T$, there exists a positive integer $J$, independent of the arbitrary point $P$ of $\mathcal{T}^{\prime}$, such that for any point $P$ in $\mathcal{T}^{\prime}$, and for any integral $j$ and $k$ such that $j>J$ and $k>J$, we have the following inequalities:

$$
\begin{aligned}
& \left|U_{j}(P)-U_{k}(P)\right|<\epsilon / 3, \\
& \left|U_{j}(P)-U_{j}(P)\right|<\epsilon / 3 \text { and }\left|U_{k}(P)-U_{k}(P)\right|<\epsilon / 3 .
\end{aligned}
$$

Hence, throughout $\mathcal{T}^{\prime}$,

$$
\left|U_{j}(P)-U_{k}(P)\right|<\epsilon \text {, if } j>J \text { and } k>J .
$$

Since the quantity $\epsilon$ was originally chosen arbitrarily, we infer that the sequence $\left\{U_{i}(P)\right\}$ converges in $T$ (uniformly in $\tau^{\prime}$ ) to a function $U(P)$. Furthermore, any given point of $\mathcal{T}^{\prime}$ is the center of a sphere within which the functions of a suitably chosen infinite subsequence of $\left\{U_{i}(P)\right\}$ form a uniformly convergent sequence of harmonic functions. Hence $U(P)$ is harmonic in $T$. Moreover, from the relations

$$
\left|U_{i}(P)-U_{i}(P)\right|<\epsilon / 3 \quad(i=0,1,2, \cdots),
$$

we see that

$$
|U(P)-U(P)| \leqq \epsilon / 3,
$$

whence we readily infer that $U(P)$ is independent of $\left\{T_{i}\right\}$.

We shall now show that $U(P)$ is unaltered if $F(P)$ is replaced by another function $F^{\prime}(P)$ which satisfies the same hypotheses as those imposed on $F(P)$. Let $U^{\prime}(P)$ be the limit of the sequence of functions $\left\{U_{i}^{\prime}(P)\right\}$ associated with $T,\left\{T_{i}\right\}$, and $F^{\prime}(P)$. Then

$$
\left\{U_{i}^{\prime}(P)-U_{i}(P)\right\}: U_{0}^{\prime}(P)-U_{0}(P), U_{1}^{\prime}(P)-U_{1}(P), U_{2}^{\prime}(P)-U_{2}(P), \cdots
$$

is the sequence of functions associated with $T,\left\{T_{i}\right\}$ and $F^{\prime}(P)-F(P)$. Since the limit of this sequence is independent of $\left\{T_{i}\right\}$ we may assume, without 
loss of generality, that the finite domains $T_{i}$ are normal. Since the function $F^{\prime}(P)-F(P)$ approaches the value zero continuously on $t$, we then have a situation in which the earlier theory of the sequence solution for continuous boundary values is applicable. The function $F^{\prime \prime}(P)=0$ approaches continuously the same boundary values on $t$ as does $F^{\prime}(P)-F(P)$, and so may be substituted for $F^{\prime}(P)-F(P)$ without affecting the limit of the sequence of functions.* Hence $\left\{U_{i}^{\prime}(P)-U_{i}(P)\right\}$ converges to zero, and so $U^{\prime}(P)=U(P)$. This completes the proof of the theorem.

Corollary 1. If there exists a solution of the Dirichlet problem, in the pseudo-classical sense, for the domain $T$ and given bounded and uniformly pseudo-continuous boundary values $f(\gamma)$ on $t$, then this solution coincides with the function $U(P)$ obtained by the method described in Theorem 21.

We may use this solution as the function $F(P)$. Then $U_{i}(P)=F(P)$, $i=0,1,2, \cdots$. Hence $U(P)=F(P)$.

COROLLARY 2. The sequence solution of the generalized Dirichlet problem $\dagger$, for the finite domain $T$ of three-dimensional space and continuous boundary values $\phi(p)$, coincides in $T$ with the function $U(P)$ (obtained by the method described in Theorem 21) corresponding to boundary values defined by the function $f(\gamma)$ which has at each element $\gamma$ of $t$ the same value as does $\phi(p)$ at the point $p$ contained in $\gamma$.

The proof is immediately obvious.

Definition 30. The function $U(P)$ the existence of which has been established in the proof of Theorem 21 is called the sequence solution of the extended Dirichlet problem for the finite domain $T$ and the boundary values $f(\gamma)$.

\section{Pseudo-Regularity $\ddagger$}

Definition 31. A function $V\left(\gamma_{1}, P\right)$ of the variable point $P$ of $T$ is said to be a pseudo-barrier for $T$ at a fixed element $\gamma_{1}$ of $t$ if the following conditions are satisfied:

(1) $V\left(\gamma_{1}, P\right)$ is a continuous superharmonic function of $P$ in $T$.

(2) $V\left(\gamma_{1}, P\right)$ approaches the value zero pseudo-continuously at $\gamma_{1}$.

(3) In the part of $T$ outside any given pseudo-spherical domain (not identical with $T$ ) corresponding to $\gamma_{1}$, the function $V\left(\gamma_{1}, P\right)$ has a positive lower bound.

* See O. D. Kellogg, loc. cit., Theorem II, p. 325.

$\dagger$ By the generalized Dirichlet problem is meant the form of the Dirichlet problem in which the sequence solution in the form developed by Wiener or Kellogg (loc. cit.) is valid.

$\ddagger$ Cf. Kellogg, loc. cit., pp. 326-328. 
Definition 32. An element $\gamma_{1}$ of $t$ is said to be pseudo-regular if there exists a pseudo-barrier for $T$ at $\gamma_{1}$.

THEOREM 22. Let $T^{(1)}$ and $T^{(2)}$ be two finite domains which have equivalent boundary elements $\gamma^{(1)}$ and $\gamma^{(2)}$ respectively. If $\gamma^{(1)}$ is a pseudo-regular boundary element of $T^{(1)}$, then $\gamma^{(2)}$ is a pseudo-regular boundary element of $T^{(2)}$.

From Definition 12 we know that there exists an infinite sequence $G_{1}, G_{2}, G_{3}, \ldots$ of common partial domains of $T^{(1)}$ and $T^{(2)}$ such that the corresponding finite closed regions form a monotone sequence of closed partial regions determining the boundary elements $\gamma^{(1)}$ of $T^{(1)}$ and $\gamma^{(2)}$ of $T^{(2)}$. Now if a boundary point $q$ of $G_{2}$ belongs to the boundary of $T^{(1)}$, then $q$ belongs to the boundary of $T^{(2) '}$. Otherwise $q$ would be an auxiliary boundary point of $G_{1}^{\prime}$ and of $G_{2}^{\prime}$ with respect to $T^{(2)}$. But this is impossible, inasmuch as the sequence $G_{1}^{\prime}, G_{2}^{\prime}, G_{3}^{\prime}, \ldots$ is monotone with respect to $T^{(2) '}$.

Let $V_{1}\left(\gamma^{(1)}, P\right)$ be a pseudo-barrier for $T^{(1)}$ at $\gamma^{(1)}$, and let $\beta$ be the greatest lower bound of $V_{1}\left(\gamma^{(1)}, P\right)$ on the proper point set $T^{(1)}-G_{2}$. The function $V_{1}\left(\gamma^{(1)}, P\right)$ is defined at every point of $T^{(2)}$ which lies on the boundary of $G_{2}$ and at such a point $V_{1}\left(\gamma^{(1)}, P\right) \geqq \beta$.

We now define a function $V_{2}\left(\gamma^{(2)}, P\right)$ in $T^{(2)}$ :

(1) If $P$ is in $G_{2}$, then $V_{2}\left(\gamma^{(2)}, P\right)$ is the smaller of the numbers $V_{1}\left(\gamma^{(1)}, P\right)$ and $\beta$, or their common value if $V_{1}\left(\gamma^{(1)}, P\right)=\beta$.

(2) If $P$ is in $T^{(2)}-G_{2}$, then $V_{2}\left(\gamma^{(2)}, P\right)=\beta$.

Now $V_{2}\left(\gamma^{(2)}, P\right)$ is a pseudo-barrier for $T^{(2)}$ at $\gamma^{(2)}$. It is clear that $V_{2}\left(\gamma^{(2)}, P\right)$ is continuous and superharmonic in $T^{(2)}$. Moreover, from Theorem 5 it follows that for sufficiently small positive $\bar{\delta}$, the pseudo-spherical domain $\mathfrak{S}\left(\gamma^{(2)}, \bar{\delta}\right)$ of $T^{(2)}$ is contained in $G_{2}$. Consequently

$$
V_{2}\left(\gamma^{(2)}, P\right) \leqq V_{1}\left(\gamma^{(1)}, P\right),
$$

in $\subseteq\left(\gamma^{(2)}, \bar{\delta}\right)$. From this relation, and from the fact that for sufficiently small positive $\delta$ the pseudo-spherical domains $\subseteq\left(\gamma^{(1)}, \delta\right)$ of $T^{(1)}$ and $\subseteq\left(\gamma^{(2)}, \delta\right)$ of $T^{(2)}$ are identical sets of points, we infer that $V_{2}\left(\gamma^{(2)}, P\right)$ approaches the value zero at the boundary element $\gamma^{(2)}$ of $T^{(2)}$, and that in the part of $T^{(2)}$ outside any given pseudo-spherical domain of $T^{(2)}$ (not identical with $T^{(2)}$ )corresponding to $\gamma^{(2)}$, the function $V_{2}\left(\gamma^{(2)}, P\right)$ has a positive lower bound.

From the existence of the pseudo-barrier $V_{2}\left(\gamma^{(2)}, P\right)$ we conclude that $\gamma^{(2)}$ is a pseudo-regular boundary element of $T^{(2)}$.

COROLLARY. The answer to the question whether or not a given boundary element $\gamma_{1}$ of $T$ is pseudo-regular depends only on the character of that part of $t$ which also belongs to the boundary of a pseudo-spherical domain, of arbitrarily small preassigned radius, corresponding to $\gamma_{1}$. 
For such a pseudo-spherical domain has a boundary element $\bar{\gamma}$ equivalent to the boundary element $\gamma_{1}$ of $T$.

THEOREM 23. Let $\gamma_{1}$ be any fixed boundary element of a given finite domain $T$, and let $U(P)$ be the sequence solution of the extended Dirichlet problem for $T$ and arbitrary bounded and uniformly pseudo-continuous boundary values $f(\gamma)$. Then a necessary and sufficient condition that every such function $U(P)$ approach with pseudo-continuity at $\gamma_{1}$ the corresponding boundary value $f\left(\gamma_{1}\right)$ is that the element $\gamma_{1}$ be pseudo-regular.

We shall first establish the necessity of the given condition. For this purpose let $U(P)$ be the sequence solution of the extended Dirichlet problem for $T$ and the boundary values $\psi(\gamma)$ determined on $t$ by the function $\Psi\left(\gamma_{1}, P\right)$ representing the pseudo-distance from $\gamma_{1}$ to the point $P$ of $T$. We shall prove that the given condition is necessary by showing that if, in this particular case, the function $U(P)$ approaches pseudo-continuously the value $\psi\left(\gamma_{1}\right)$ at the boundary element $\gamma_{1}$, then $U(P)$ is a pseudo-barrier for $T$ at $\gamma_{1}$.

Let $\left\{T_{i}\right\}$ be any pseudo-normal sequence of domains in $T$. The function $U(P)$ may be obtained as the limit of the sequence of functions $\left\{U_{i}(P)\right\}$ associated with $T,\left\{T_{i}\right\}$, and $\Psi\left(\gamma_{1}, P\right)$. Now $\Psi\left(\gamma_{1}, P\right)$ is subharmonic in $T$, and so $\left\{U_{i}(P)\right\}$ is a monotone increasing sequence, and

$$
U(P) \geqq \Psi\left(\gamma_{1}, P\right),
$$

throughout $T$. That $U(P)$ is a pseudo-barrier for $T$ at $\gamma_{1}$ is now obvious from the properties of pseudo-distance given in Theorem 20 .

In order to establish the sufficiency of the given condition, we now let $U(P)$ be the sequence solution of the extended Dirichlet problem for $T$ and arbitrarily given bounded and uniformly pseudo-continuous boundary values, $f(\gamma)$. We shall show that if there exists a barrier, $V\left(\gamma_{1}, P\right)$, for $T$ at a given element $\gamma_{1}$ of $t$, then to any preassigned positive $\epsilon$ there corresponds a positive $\delta$ such that

$$
\left|U(P)-f\left(\gamma_{1}\right)\right|<\epsilon
$$

throughout $\mathfrak{S}\left(\gamma_{1}, \delta\right)$. We may assume, without loss of generality, that $V\left(\gamma_{1}, P\right)$ is bounded* in $T$.

Let $F(P)$ be any function which is bounded and continuous in $T$, and approaches the boundary values $f(\gamma)$ with uniform pseudo-continuity. $\dagger$ There exist positive constants $\bar{\delta}, B$, and $b$ such that

\footnotetext{
* The existence of a pseudo-barrier for $T$ at $\gamma_{1}$ implies the existence of a bounded pseudo-barrier for $T$ at $\gamma_{1}$, as may be readily seen from the discussion of Theorem 22 .

$\dagger$ The continuity of $F(P)$ in $T$ is necessarily pseudo-uniform, by Theorem 8 .
} 


$$
\begin{array}{cl}
\left|F(P)-f\left(\gamma_{1}\right)\right|<\frac{\epsilon}{2}, & \text { in } \mathfrak{S}\left(\gamma_{1}, \bar{\delta}\right), \\
b<V\left(\gamma_{1}, P\right), & \text { in } T-\mathfrak{S}\left(\gamma_{1}, \bar{\delta}\right),
\end{array}
$$

and

$$
\left|F(P)-f\left(\gamma_{1}\right)\right|<B b, \quad \text { in } T-\mathfrak{S}\left(\gamma_{1}, \bar{\delta}\right) .
$$

From these inequalities we infer that

$$
\left|F(P)-f\left(\gamma_{1}\right)\right|<B V\left(\gamma_{1}, P\right), \quad \text { in } T-\Im\left(\gamma_{1}, \bar{\delta}\right),
$$

and

$$
\left|F(P)-f\left(\gamma_{1}\right)\right|<B V\left(\gamma_{1}, P\right)+\frac{\epsilon}{2}, \quad \text { in } T .
$$

Let $\left\{T_{i}\right\}$ be any pseudo-normal sequence of domains in $T$, and let $\left\{U_{i}(P)\right\}$ be the sequence of functions associated with $T,\left\{T_{i}\right\}$, and $F(P)$. From the Corollary of Theorem 10, we know that given any positive $\epsilon^{\prime}$ and any non-negative integer $i$, there exists a positive $\delta_{i}^{\prime}$, independent of $P$, such that

$$
-\epsilon^{\prime}<U_{i}(P)-U_{i+1}(P),
$$

at each point $P$ of $T_{i+1}$ at a distance less than $\delta_{i}^{\prime}$ from the boundary of $T_{i+1}$. Suppose now that for each point $P$ of $T$ we have also, for some such $i$,

$$
0 \leqq\left[f\left(\gamma_{1}\right)+B V\left(\gamma_{1}, P\right)+\frac{\epsilon}{2}\right]-U_{i}(P),
$$

a relation which is already known to be valid when $i=0$. Then for each point $P$ of $T_{i+1}$ at a distance less than $\delta_{i}^{\prime}$ from the boundary of $T_{i+1}$ we have

$$
-\epsilon^{\prime}<\left[f\left(\gamma_{1}\right)+B V\left(\gamma_{1}, P\right)+\frac{\epsilon}{2}\right]-U_{i+1}(P) .
$$

From Theorem 17 we infer that

$$
0 \leqq\left[f\left(\gamma_{1}\right)+B V\left(\gamma_{1}, P\right)+\frac{\epsilon}{2}\right]-U_{i+1}(P),
$$

throughout $T_{i+1}$, and so throughout $T$. By mathematical induction we conclude that for every non-negative integer $i$,

$$
0 \leqq\left[f\left(\gamma_{1}\right)+B V\left(\gamma_{1}, P\right)+\frac{\epsilon}{2}\right]-U_{i}(P),
$$

throughout $T$. 
By allowing $i$ to become infinite in this relation we see that

$$
U(P) \leqq f\left(\gamma_{1}\right)+B V\left(\gamma_{1}, P\right)+\frac{\epsilon}{2},
$$

throughout $T$. By analogous reasoning we infer that

$$
U(P) \geqq f\left(\gamma_{1}\right)-B V\left(\gamma_{1}, P\right)-\frac{\epsilon}{2},
$$

throughout $T$. Now there exists a positive $\delta$ such that

$$
0<V\left(\gamma_{1}, P\right)<\frac{\epsilon}{2 B}
$$

throughout $\subseteq\left(\gamma_{1}, \delta\right)$. Hence we have

$$
\left|U(P)-f\left(\gamma_{1}\right)\right|<\epsilon,
$$

throughout $\subseteq\left(\gamma_{1}, \delta\right)$. This relation establishes the sufficiency of the given condition.

COROLlARY. If $T$ is an ordinary finite domain and if each boundary element of $T$ is pseudo-regular, then $T$ is pseudo-normal.

The validity of this proposition is readily established by the use of Theorems 12 and 23.

TheOREM 24. If the point $p$ contained in a given boundary element $\gamma$ of $T$ is a regular boundary point of some partial domain $G$ of $T$ corresponding to a closed partial region of $T^{\prime}$ which contains $\gamma$, then $\gamma$ is a pseudo-regular boundary element of $T$.

We may choose a positive number $\rho_{1}$ such that $\subseteq\left(\gamma, \rho_{1}\right)$ is a proper subset of $G$. If $\rho_{1}>\rho_{2}>\rho_{3}>\cdots, \rho_{i} \rightarrow 0$, then $\mathfrak{S}^{\prime}\left(\gamma, \rho_{1}\right), \mathfrak{S}^{\prime}\left(\gamma, \rho_{2}\right), \mathfrak{S}^{\prime}\left(\gamma, \rho_{3}\right), \cdots$ determines $\gamma$. Let $U(p, P)$ be a barrier for $G$ at $p$. For each $i>1$ let $\beta_{i}$ be the greatest lower bound of $\mathcal{U}(p, P)$ in $G-G \cdot S_{i}$, where $S_{i}$ is the interior of the sphere of radius $\rho_{i}$ with center at $p$. We now define $V_{i}(\gamma, P), i=2,3$, $4, \cdots$, by requiring that

(1) if $P$ is in $\subseteq\left(\gamma, \rho_{i}\right)$ then $V_{i}(\gamma, P)$ is the smaller of $U(p, P)$ and $\beta_{i}$, or their common value if $U(p, P)=\beta_{i}$;

(2) if $P$ is in $T-\Im\left(\gamma, \rho_{i}\right)$ then $V_{i}(\gamma, P)=\beta_{i}$.

For fixed $\gamma$ each of the functions $V_{i}(\gamma, P)$ is continuous and superharmonic in $T$, and $0<V_{i}(\gamma, P) \leqq \beta_{2}, i=2,3,4, \cdots$. Hence

$$
V(\gamma, P)=\sum_{i=2}^{\infty} 2^{-i} V_{i}(\gamma, P)
$$


is continuous and superharmonic in $T$, inasmuch as the series is uniformly convergent in $T$. Now in $\subseteq\left(\gamma, \rho_{2}\right)$

$$
V(\gamma, P) \leqq U(p, P) \cdot \sum_{i=2}^{\infty} 2^{-i}
$$

Hence (considered as a function of $P) V(\gamma, P)$ approaches the value zero pseudo-continuously at $\gamma$. On the other hand, given any positive $\rho$, we can choose $k$ so that $\subseteq\left(\gamma, \rho_{k}\right)$ is a subset of $\mathfrak{S}(\gamma, \rho)$. In $T-\mathfrak{S}(\gamma, \rho)$,

$$
V(\gamma, P) \geqq \sum_{i=k}^{\infty} 2^{-i} \beta_{i}>0 .
$$

Hence $V(\gamma, P)$ is a pseudo-barrier for $T$ at $\gamma$, and the theorem is proved.

We now consider two lemmas*:

Lemma 1. Let $T$ be a finite normal domain. Then to each point $p$ of $t$ there corresponds a function $W(p, P)$ of a variable point $P$ of $T$ having the following properties:

(1) $W(p, P)$ is bounded and harmonic in $T$.

(2) Given any positive $\epsilon$ there exists a positive $\delta$, independent of $p$, such that if $\overline{p P}<\delta$ then $|W(p, P)|<\epsilon$.

(3) $W(p, P) \geqq \bar{p}$, throughout $T$.

For fixed $p$ we take $W(\underline{p}, P)$ as the solution of the Dirichlet problem with boundary values $w(p, q)=\overline{p q}$ on $t$. Then $W(p, P)-\overline{p P}$ is superharmonic in $T$, and approaches the boundary value zero continuously on $t$. Hence items (1) and (3) of the conclusion of the lemma are valid.

Given any point $p_{1}$, of $t$, there exists a positive $\delta\left(p_{1}\right)$ such that $W\left(p_{1}, P\right)$ $<\epsilon / 2$ for each point $P$ of $T$ such that $\overline{p_{1} P}<\delta\left(p_{1}\right)$. Furthermore,

$$
W(p, P) \leqq W\left(p_{1}, P\right)+\overline{p p_{1}}
$$

for any point $P$ of $T$ and any pair of points $p$ and $p_{1}$ on $t$. This follows from the fact that $W(p, P)-W\left(\underline{p_{1}}, P\right)$ is harmonic in $T$ and approaches continuously the boundary value $\overline{p q}-\overline{p_{1} q}$ at the arbitrary point $q$ of $t$. Hence for $\overline{p p_{1}}<\epsilon / 2$ and $\overline{p_{1} P}<\delta\left(p_{1}\right)$ we have

$$
0<W(p, P)<\epsilon .
$$

This implies that each point $p_{1}$ of the bounded closed set $t$ is the center of a sphere $S\left(p_{1}\right)$ such that if $P$ and $p$ are any points of $T$ and $t$ respectively

* I wish to thank Professor J. J. Gergen for many helpful suggestions in the revision of this paper, particularly in connection with these lemmas and their application to Theorem 25 , which was originally stated and proved only for ordinary domains. 
which lie in the interior of the same $S\left(p_{1}\right)$ then $|W(p, P)|<\epsilon$. By the HeineBorel Theorem there exists a finite set of overlapping spheres of this type enclosing $t$. There exists a positive constant $\delta$, independent of $p$, such that a sphere of radius $\delta$ with center at any point of $t$ lies entirely in the interior of some sphere of this finite set. For this $\delta$ the assertion given in item (2) of the conclusion of the lemma is valid.

LEMma 2. Let $T$ be a finite normal domain. Then to each element $\gamma$ of there corresponds a function $V(\gamma, P)$ of a variable point $P$ of $T$ having the following properties:

(1) $V(\gamma, P)$ is bounded, continuous and superharmonic in $T$.

(2) Given any positive $\epsilon$, there exists a positive $\delta$, independent of $\gamma$, such that $|V(\gamma, P)|<\epsilon$ for every $P$ in $\subseteq(\gamma, \delta)$.

(3) Given any $\rho$ such that $0<\rho<\bar{\rho}$ (where $2 \bar{\rho}$ is the diameter of $T$ ) there exists $a$ positive $b(\rho)$, independent of $\gamma$ and $P$, such that $b(\rho) \leqq V(\gamma, P)$ for every point $P$ in the proper set $T-\Im(\gamma, \rho)$.

Choose $\bar{\rho}>\rho_{1}>\rho_{2}>\rho_{3}>\cdots$, so that $\rho_{i}$ tends to zero. We now define a set of functions $V_{i}(\gamma, P), i=1,2,3, \cdots$, for any $\gamma$ of $t$ and any $P$ of $T$ by making the following requirements:

(1) If $P$ is in $\varsigma\left(\gamma, \rho_{i}\right)$ then $V_{i}(\gamma, P)$ is the smaller of $W(p, P)$ and $\rho_{i}$ (where $p$ is the point contained in $\gamma$ ) or their common value if $W(p, P)=\rho_{i}$.

(2) If $P$ is in $T-\Im\left(\gamma, \rho_{i}\right)$, then $V_{i}(\gamma, P)=\rho_{i}$.

For fixed $\gamma$, each $V_{i}(\gamma, P)$ is bounded, continuous and superharmonic in $T$. Moreover, $0<V_{i}(\gamma, P) \leqq \rho_{1}$, throughout $T$. Hence, for each $\gamma$ of $t$,

$$
V(\gamma, P)=\sum_{i=1}^{\infty} 2^{-i} V_{i}(\gamma, P)
$$

is continuous and superharmonic in $T$; also,

$$
0<V(\gamma, P) \leqq W(p, P),
$$

throughout $T$. Hence items (1) and (2) of the conclusion of the lemma are valid. Moreover, given any $\rho$ such that $0<\rho<\bar{\rho}$, we can choose, independently of $\gamma$, an integer $k$ such that $\subseteq\left(\gamma, \rho_{k}\right)$ is contained in the partial domain $\mathfrak{S}(\gamma, \bar{\rho})$ of $T$. We have then in $T-\mathfrak{S}(\gamma, \rho)$,

$$
V(\gamma, P) \geqq \sum_{i=k}^{\infty} 2^{-i} \rho_{i} .
$$

Choosing $b(\rho)$ as the value of the series given in this relation, we see that item (3) of the conclusion of the lemma is valid.

Theorem 25. If $T$ is a normal finite domain, then $T$ is pseudo-normal. 
Let $U(P)$ be the sequence solution of the extended Dirichlet problem for the normal finite domain $T$ and arbitrarily assigned bounded and pseudouniformly continuous boundary values $f(\gamma)$. We need to show that given any positive $\epsilon$, there exists a positive $\delta$ independent of the arbitrarily chosen element $\gamma_{1}$ of $t$, such that

$$
\left|U(P)-f\left(\gamma_{1}\right)\right|<\epsilon
$$

throughout $\mathfrak{S}\left(\gamma_{1}, P\right)$.

Let $F(P)$ be any function which is bounded and continuous in $T$ and approaches with uniform pseudo-continuity the boundary values $f(\gamma)$ on $t$. We may then choose, independently of $\gamma_{1}$, a positive quantity $\bar{\delta}$ less than half the diameter of $T^{\prime}$ such that in $\subseteq\left(\gamma_{1}, \bar{\delta}\right)$

$$
\left|F(P)-f\left(\gamma_{1}\right)\right|<\epsilon / 2 .
$$

Let us now consider again the reasoning by which we established the sufficiency of the condition given in Theorem 23. Since $T$ is now assumed to be normal we may use as a pseudo-barrier $V\left(\gamma_{1}, P\right)$ the function introduced in Lemma 2. We know then that there exist constants $b(\bar{\delta})$ and $B(\bar{\delta})$, independent of $\gamma_{1}$, such that

$$
b(\bar{\delta})<V\left(\gamma_{1}, P\right), \quad \text { in } T-\mathfrak{S}\left(\gamma_{1}, \bar{\delta}\right),
$$

and

$$
\left|F(P)-f\left(\gamma_{1}\right)\right|<B(\bar{\delta}) b(\bar{\delta}), \quad \text { in } T-\mathfrak{S}\left(\gamma_{1}, \bar{\delta}\right) .
$$

Hence,

$$
\left|F(P)-f\left(\gamma_{1}\right)\right|<B(\bar{\delta}) V\left(\gamma_{1}, P\right)+\epsilon / 2, \quad \text { in } T .
$$

By the same reasoning as that used in the proof of Theorem 23, we infer that

$$
\left|U(P)-f\left(\gamma_{1}\right)\right|<B(\bar{\delta}) V\left(\gamma_{1}, P\right)+\epsilon / 2,
$$

throughout $T$. Again using Lemma 2, we may choose a positive constant $\delta$, independent of $\gamma_{1}$, such that

$$
0<V\left(\gamma_{1}, P\right)<\frac{\epsilon}{2 B(\bar{\delta})},
$$

in $\mathfrak{S}\left(\gamma_{1}, \delta\right)$. Hence

$$
\left|U(P)-f\left(\gamma_{1}\right)\right|<\epsilon,
$$

in $\subseteq\left(\gamma_{1}, \delta\right)$ : This completes the proof of the theorem.

Dartmouth College,

HANOVER, N. H. 